\title{
A RECURSION-THEORETIC CHARACTERIZATION OF THE RAMIFIED ANALYTICAL HIERARCHY
}

\author{
BY \\ RICHARD BOYD, GUSTAV HENSEL AND HILARY PUTNAM
}

Introduction. According to a classical theorem of Post, the arithmetical sets may be obtained by the following construction:

Step 0: "take" all the r.e. sets.

Step $n+1$ : "add" all sets which are r.e. in sets "taken" at a previous stage.

Moreover, this construction is intimately related to the Kleene arithmetical hierarchy, defined in terms of the number and quality of alternating numberquantifiers needed to define a set (using a matrix which is a recursive predicate of integers).

In terms of degrees of unsolvability as opposed to sets, what this construction amounts to is:

Step $n(n=0,1,2, \ldots)$ : "take" all sets recursively enumerable in sets of the degree $j^{(n)}(0)$.

[Here $j$ denotes the ordinary jump operator on degrees, $j^{(0)}(0)=0, j^{(n+1)}(0)$ $=j\left(j^{(n)}(0)\right)$.]

If $\Gamma$ is a collection of degrees, we write $R U(\Gamma)$ for the collection of all sets recursive in sets whose degrees are in $\Gamma . R U(\Gamma)$ is the smallest class of sets containing all sets whose degrees are in $\Gamma$ and closed under "recursive in".

The arithmetical hierarchy is thus represented by the linear sequence of degrees 0 , $j(0), j j(0), \ldots, j^{(n)}(0), \ldots$ in the sense that the successive collections

$$
R U\left(\left\{0, j(0), \ldots, j^{(n)}(0)\right\}\right)
$$

(as $n=1,2, \ldots$ ) are just the successive levels of that hierarchy.

In extending the hierarchy into the transfinite, we seek an increasing function $d$ from the ordinals in some section of the countable ordinals into degrees, such that:

(1) $d(0)=0$.

(2) $d(\alpha+1)=j(d(\alpha))$.

(3) $d(\lambda)$ is in some sense a least (or possibly an "almost least" if no "least" exists) bound on $R U(\{d(\alpha) \mid \alpha<\lambda\})$. (For all limit numbers $\lambda$ in the appropriate section.)

The difficulty in this undertaking lies with clause (3). By a theorem of Spector, the $\omega$-sequence of degrees $0, j(0), j j(0), \ldots, j^{(n)}(0), \ldots$ has no least upper bound (indeed no $\omega$-sequence of increasing degrees has a least upper bound). Thus $d(\lambda)$ cannot literally be a least upper bound on $R U(\{d(\alpha) \mid \alpha<\lambda\})$, even when $\lambda=\omega$.

Received by the editors June 20, 1966. 
Thus we are led to seek further conditions to impose on the choice of $d(\lambda)$ which will make that choice reasonably unique.

Let $\Gamma$ again be a collection of degrees. We shall say that a degree $d$ is a uniform upper bound (u.u.b.) on $R U(\Gamma)$ if the class $R U(\Gamma)$ is an $A$-r.e. class of $A$-recursive sets, where $A$ is any member of $d$. What this means is that the sets of $R U(\Gamma)$ are not just individually recursive in $A$, but that the whole collection is uniformly recursive in $A$, in the sense that the members of $R U(\Gamma)$ may be "Gödel numbered" in at least one way such that questions of the form "does the integer $n$ belong to the set with the Gödel number $m$ ?" may be effectively answered using $A$ as an oracle.

It is clear that if one $A$ in a degree $d$ (recall that a degree is an equivalence class of sets) has the property just stated, so does any other member of $d$ : thus the property of being a u.u.b. is a property of the degree, even though we define it in terms of an arbitrary representative.

The standard extensions of the arithmetical hierarchy due to Davis, Mostowski, Kleene, etc., all have the property that the degree $d_{\lambda}$ they associate with any limit ordinal $\lambda$ is not just an upper bound, but a uniform upper bound, on $R U\left(\left\{d_{\alpha} \mid \alpha<\lambda\right\}\right)$, where $d_{\alpha}$ is the degree associated with $\alpha$ in the particular hierarchy.

This suggests modifying clause (3) to read:

(3)* $d(\lambda)$ is a least u.u.b. on $R U(\{d(\alpha) \mid \alpha<\lambda\})$.

Unfortunately, this does not quite work. However, it almost "works" in the following sense: Call a degree $d_{1}$ an $n$-least u.u.b. on a class $S$ of sets if the following two conditions are fulfilled:

(a) $d_{1}$ is a u.u.b. on $S$.

(b) If $d_{2}$ is any other u.u.b. on $S, d_{1} \leqq_{T} d_{2}^{(n)}$.

Then modify clause (3) to read:

(3) $\dagger d(\lambda)$ is an n-least u.u.b. on $R U(\{d(\alpha) \mid \alpha<\lambda\})$, for some $n$.

[N.B. This does not guarantee a unique choice of $d(\lambda)$, but any two choices $d_{1}, d_{2}$ at the same $\lambda$ will at least be arithmetical in one another.]

A function $d$ satisfying (1), (2), and (3) $\dagger$ will be called an admissible degree hierarchy. The question which motivated the present paper is: how far do admissible degree hierarchies extend?

The answer, surprisingly, depends on the notion of a ramified analytical set. The ramified analytical sets of integers are just the sets definable in predicative analysis, provided the cumulative "orders" of predicative analysis are extended into the transfinite through the classical ordinals (a precise definition occurs later in this paper). Paul Cohen showed in [C] that there is a countable ordinal, here called $\beta_{0}$, such that no new sets are obtained in predicative analysis past the "order" $\beta_{0}$. (In other words, if $A_{\alpha}$ is the $\alpha$ th "order", every ramified analytical set belongs to some $A_{\alpha}$ with $\alpha<\beta_{0}$.)

The ramified analytical sets thus have a certain analogy to the "constructible" sets of Gödel [G]. However, $A_{\alpha+1}$ consists of just the sets of integers definable over $A_{\alpha}$ by a formula of 2-N.T. (2nd order number theory), whereas $M_{\alpha+1}$ (the 
$(\alpha+1)$ st "order" in predicative set theory, or the hierarchy of "constructible sets") consists of all sets (not just the sets of integers) definable over $M_{\alpha}$ by a formula of set theory. (The difference is crucial, as there is no ordinal $\alpha$ such that all constructible sets belong to $M_{\alpha}$, whereas there is an ordinal $\alpha$-namely $\beta_{0}$-such that all ramified analytical sets belong to $A_{\alpha}$.)

The main result of the present paper is that there is an admissible degree hierarchy defined on $\Sigma\left(\beta_{0}\right)$, the section of the ordinals by the ordinal $\beta_{0}$, and no admissible degree hierarchy defined on $\Sigma\left(\beta_{0}+1\right)$. Moreover, the ramified analytical sets are precisely the sets for which we can obtain degrees of unsolvability in an admissible degree hierarchy.

In future papers we plan to show that this result is "best possible" in the sense that the "for some $n$ " in clause (3) cannot be replaced by any fixed $n$ without reducing the ordinal at which there ceases to be a hierarchy. This phenomenon will be shown to be connected with the existence of ordinals $\beta^{(n)}<\beta_{0}$ at which $A_{\beta}(n)$ is a model for the $n$-quantifier comprehension axioms of 2-N.T. (for each fixed $n$ ).

We also plan to show (in joint work with Joan Lukas) that there are arithmetically-minimal systems of notations containing notations for all ordinals $<\beta_{0}$, but no arithmetically-minimal system containing a notation for $\beta_{0}$ itself. [The notion of a minimal system was defined by Enderton in [E]. The definition of arithmetically-minimal is similar, with "arithmetical in " replacing "recursive in".] It is striking that two different approaches to the problem of extending the arithmetical hierarchy - the approach via "arithmetically-minimal systems of notations" and the approach via "n-least u.u.b."-lead to the same "stopping point" $\beta_{0}$.

Paul Cohen conjectured in $[\mathbf{C}]$ that the ramified analytical sets form the minimal $\beta$-model for analysis. We include a proof of this result (which follows easily from our main theorems) as a final section of this paper. This result was first obtained independently by Gandy and by Putnam who plan to publish a joint paper [GP].

Notations not specially explained are standard recursive function theory or logic. We write $|a|_{0}$ for the ordinal for which $a$ is a "notation", when $a \in O$.

1. Admissible degree-hierarchies. Kleene and Davis [D] extend the arithmetical hierarchy by assigning a set $H_{a}$ to each ordinal notation $a$ from the Church-Kleene system, $S_{3}$, by the following induction:

(a) $H_{1}=\varnothing$.

(b) If $2^{a} \in O$, then $H_{2^{a}}=j\left(H_{a}\right)=\left\{x \mid(\exists z) T^{H_{a}}(x, x, z)\right\}$.

(c) If $3 \cdot 5^{e} \in O$, then $H_{3 \cdot 5^{e}}=\left\{x \mid(x)_{0} \in H_{e_{(x)}}\right\}$, where, in general, $e_{y}=\{e\}\left(y_{0}\right)$ where $0_{0}=1$ and $(n+1)_{0}=2^{n_{0}}$.

By the Spector "uniqueness" theorem [S], we know that if $a, b \in 0$, and $|a|_{0}$ $=|b|_{0}$, then $H_{a} \equiv_{T} H_{b}$; thus the hyperarithmetical hierarchy is an ordinal hierarchy of degrees of unsolvability. Let $D_{\text {H.A. }}$ be the function on $\Sigma\left(\omega_{1}\right)=$ the section of the ordinals by $\omega_{1}$ (the first nonrecursive ordinal) such that $D_{\text {H.A. }}(\alpha)=\operatorname{deg}\left(H_{a}\right)$, where $a \in O$ and $\alpha=|a|_{0}$. 
Definition 1.1. Let $\Gamma$ be a collection of degrees; then

$$
R U(\Gamma)=\{S \mid S \text { is recursive in some element of } \Gamma\} .
$$

Definition 1.2. Let $A$ be a set and $\Delta$ be a countable collection of sets. Then $A$ is a uniform upper-bound (u.u.b.) to $\Delta$ iff $\Delta$ is an $A$-recursively enumerable collection of $A$-recursive sets, i.e., iff there is an $A$-recursive function $f$ such that the class of partial functions $\left\{\{f(n)\}^{A}\right\}_{n \in N}$ is exactly the class of (total) characteristic functions for the sets in $\Delta$.

Definition 1.3. If $\Gamma$ is a collection of degrees, $d \in \Gamma$, and $n \in N$, then $d$ is an n-least member of $\Gamma$ iff $d$ is recursive in $j^{(n)}(e)$ for all $e \in \Gamma$, where $j$ represents the jump operator and we take $j^{(0)}(e)=e$.

The function $D_{\text {H.A. }}$ on $\Sigma\left(\omega_{1}\right)$ has the following properties:

(i) $D_{\text {H.A. }}(0)=\operatorname{deg}(\varnothing)$.

(ii) $D_{\text {H.A. }}(\alpha+1)=j\left(D_{\text {H.A. }}(\alpha)\right)$.

(iii) If $\lambda$ is a limit ordinal, then $D_{\text {H.A. }}(\lambda)$ is a 2-least u.u.b. to $R U\left\{D_{\text {H.A. }}(\alpha) \mid \alpha<\lambda\right\}$.

[In an unpublished paper, Enderton and Putnam show that if $|e|_{0}=\lambda$, then $H_{e}$ is recursive in the jump-jump of every set in which all sets in $R U\left\{D_{\text {H.A. }}(\alpha) \mid \alpha<\lambda\right\}$ are recursive. That is, if $d$ is the degree of any upper-bound (not necessarily uniform) of $R U\left\{D_{\text {H.A. }}(\alpha) \mid \alpha<\lambda\right\}$ then $D_{\text {H.A. }}(\lambda) \leqq d^{\prime \prime}$. Thus for $\lambda<\omega_{1}, D_{\text {H.A. }}(\lambda)$ is a 2-least upper bound on $R U\left\{D_{\text {H.A. }}(\alpha) \mid \alpha<\lambda\right\}$. That $D_{\text {H.A. }}(\lambda)$ is a 2-least uniform upper bound follows from the fact that $H_{e}$ is a u.u.b. on $R U\left\{D_{\text {H.A. }}(\alpha) \mid \alpha<\lambda\right\}$ if $|e|_{0}=\lambda$. This also follows from [HP], where Hensel and Putnam showed that $D_{\text {H.A. }}(\lambda)$ is a least nice bound on $R U\left\{D_{\text {H.A. }}(\alpha) \mid \alpha<\lambda\right\}$. Here it is enough to notice that, since the predicates $\left\{\{x\}^{A}\right.$ is the jump of $\left.\{y\}^{A}\right\}$ and $\left\{\{x\}^{A}\right.$ is the cartesian product of $\{y\}^{A}$ and $\left.\{z\}^{A}\right\}$ are both expressible in 2 number quantifier form in $A$, if $A$ is a u.u.b. on any family of sets, then $j(j(A))$ is a nice bound on the same family (cf. [HP], Definition 3 and Theorems 2 and 3, pp. 72-75).]

We generalize these properties of the hyperarithmetical hierarchy to obtain the following more general notion:

Definition 1.4. Let $\beta$ be a nonzero ordinal and $D$ a function from $\Sigma(\beta)$ into the family of degrees of unsolvability. Then $D$ is an admissible degree-hierarchy (a.d.h.) on $\Sigma(\beta)$ iff:

(i) $D(0)=\operatorname{deg}(\varnothing)$.

(ii) $D(\alpha+1)=j(D(\alpha))$.

(iii) If $\lambda$ is a limit ordinal, then there exists an integer $n \in N$ such that $D(\lambda)$ is an n-least u.u.b. to $R U\{D(\alpha) \mid \alpha<\lambda\}$.

Definition 1.5. Let $D$ be a function from $\Sigma(\beta)(\beta>0)$ into the family of degrees of unsolvability, and let $S$ be any set of integers. Then $D$ is an $S$-admissible degreehierarchy iff: $D$ satisfies the conditions of Definition 1.4, with condition (i) replaced by condition (i)'

(i)' $D(0)=\operatorname{deg}(S)$.

We now establish some facts about a.d.h.'s. 
LEMMA 1.6. Suppose the following are true:

(i) $\lambda$ is a limit ordinal.

(ii) $D$ is an $S$-a.d.h. on $\Sigma(\lambda)$.

(iii) $\operatorname{deg}\left(S^{\prime}\right)$ is an n-least u.u.b. to $R U\{D(\alpha) \mid \alpha<\lambda\}$.

(iv) $D^{\prime}$ is an $S^{\prime}$-a.d.h. on $\Sigma(\beta)$ for some $\beta>0$.

Then $D^{*}$ is an $S$-a.d.h. on $\Sigma(\lambda+\beta)$, where

(a) If $0 \leqq \alpha<\lambda, D^{*}(\alpha)=D(\alpha)$.

(b) If $0 \leqq \alpha<\beta, D^{*}(\lambda+\alpha)=D^{\prime}(\alpha)$.

Proof. Obvious from Definition 1.5.

LEMMA 1.7. Let $S$ be any set of integers, and let $D_{1}$ and $D_{2}$ be $S$-a.d.h.'s on $\Sigma(\beta)$. Then for all limit ordinals $\lambda \leqq \beta$,

$$
R U\left\{D_{1}(\alpha) \mid \alpha<\lambda\right\}=R U\left\{D_{2}(\alpha) \mid \alpha<\lambda\right\} .
$$

Proof. We proceed by induction on $\lambda$. The first case to consider is $\lambda=\omega$, but $D_{1} / \omega=D_{2} / \omega$, and the result is immediate.

Now suppose that $\lambda$ is a limit ordinal, $\lambda \leqq \beta$, and the result holds for all smaller ordinals. One of two cases obtains:

(i) There is a largest limit ordinal $\lambda^{*}<\lambda$. If this is the case then, by the induction hypothesis we have

$$
R U\left\{D_{1}(\alpha) \mid \alpha<\lambda^{*}\right\}=R U\left\{D_{2}(\alpha) \mid \alpha<\lambda^{*}\right\} .
$$

Let $d_{1}=D_{1}\left(\lambda^{*}\right), d_{2}=D_{2}\left(\lambda^{*}\right)$. Then $d_{1}$ and $d_{2}$ are respectively $n_{1}$-least and $n_{2}$-least u.u.b.'s to the same set, so $d_{1} \leqq_{T} j^{\left(n_{1}\right)}\left(d_{2}\right)$ and $d_{2} \leqq_{T} j^{\left(n_{2}\right)}\left(d_{1}\right)$. But since

$$
R U\left\{D_{i}(\alpha) \mid \alpha<\lambda\right\}=R U\left\{j^{(n)}\left(d_{i}\right) \mid n \in N\right\} \quad i=1,2,
$$

it is immediate that $R U\left\{D_{1}(\alpha) \mid \alpha<\lambda\right\}=R U\left\{D_{2}(\alpha) \mid \alpha<\lambda\right\}$, as required.

(ii) $\lambda$ is a limit of limit ordinals. If this is the case then, of course

$$
R U\left\{D_{i}(\alpha) \mid \alpha<\lambda\right\}=\bigcup_{\text {Lim } \beta, \beta<\lambda} R U\left\{D_{i}(\alpha) \mid \alpha<\beta\right\} \quad i=1,2
$$

but by the induction hypothesis $R U\left\{D_{1}(\alpha) \mid \alpha<\beta\right\}=R U\left\{D_{2}(\alpha) \mid \alpha<\beta\right\}$ for all limit $\beta<\lambda$, so again we have $R U\left\{D_{1}(\alpha) \mid \alpha<\lambda\right\}=R U\left\{D_{2}(\alpha) \mid \alpha<\lambda\right\}$.

LEMMA 1.8. Let $D$ be an a.d.h. on $\Sigma\left(\omega_{1}\right)$; then $R U\left\{D(\alpha) \mid \alpha<\omega_{1}\right\}=$ H.A. $=$ the family of hyperarithmetical sets.

Proof. We already know that the a.d.h. $D_{\text {H.A. }}$ has the property that

$$
R U\left\{D_{\text {H.A. }}(\alpha) \mid \alpha<\omega_{1}\right\}=\text { H.A. }
$$

If $D$ is any other a.d.h. on $\Sigma\left(\omega_{1}\right)$ then we know from Lemma 1.7 and the fact that $\omega_{1}$ is a limit ordinal that

$$
R U\left\{D(\alpha) \mid \alpha<\omega_{1}\right\}=R U\left\{D_{\text {H.A. }}(\alpha) \mid \alpha<\omega_{1}\right\}=\text { H.A. }
$$


The following relativized form of Lemma 1.8 is also useful:

LEMMA 1.9. Let $D$ be an $S$-a.d.h. on $\Sigma\left(\omega_{1}^{S}\right) \quad\left(\omega_{1}^{S}=\right.$ the least ordinal not recursive in $S)$. Then $R U\left\{D(\alpha) \mid \alpha<\omega_{1}^{S}\right\}=H . A .^{S}=$ the family of $S$-hyperarithmetical sets.

2. The ramified analytical hierarchy. In [K] Kleene considers a family of subsets $A_{\alpha}$ of the power set of the integers defined as follows:

Definition 2.1. $A_{0}=\{S \subset N \mid S$ is arithmetical $\}$.

$A_{\alpha+1}=\left\{S \subset N \mid S\right.$ is definable over $A_{\alpha}$ in 2nd-order number theory (2-N.T.) with set constants representing sets in $\left.A_{\alpha}\right\}$.

If $\lambda$ is a limit ordinal then $A_{\lambda}=\bigcup_{\beta<\lambda} A_{\beta}$.

Kleene carries this construction only as far as constructive $\omega_{1}$ (by induction on $S_{3}$ ), but the construction obviously generalizes to all the classical ordinals. It is obvious by a cardinality argument that there must be some ordinal $\beta$ such that $A_{\beta+1}=A_{\beta}$. Let $\beta_{0}$ be the first such ordinal. In [C] P. J. Cohen showed that $\beta_{0}$ is countable (essentially by carrying out the construction of the sets $A_{\alpha}$ in a countable, transitive $\varepsilon$-model of $Z F$ set theory and showing that Definition 2.1 is "absolute" in the sense of Gödel [G]).

Definition 2.2. $S \subset N$ is ramified analytical just in case $S \in A_{\alpha}$ for some $\alpha$. We will also use "R.A." to denote the family of all ramified analytical sets.

The condition $A_{\beta_{0}+1}=A_{\beta_{0}}$ means that R.A. $=A_{\beta_{0}}$ is an $\omega$-model for analysis (i.e., R.A. satisfies all the 2 nd-order comprehension schemata). An $\omega$-model $\mathfrak{M}$ is called a $\beta$-model just in case the predicate " $(\alpha)[\alpha$ is not an infinite descending path in $R$ ]" holds of a linear ordering $R$ in $\mathfrak{M}$ only when $R$ is actually a well-ordering. Cohen conjectured in [C] that R.A. was the minimum $\beta$-model for analysis. This result has subsequently been proved independently by Gandy and Putnam [GP].

We need to establish a large number of facts about the sets $A_{\alpha}$ and about the a.d.h.'s. Some of these facts are very obvious, and we shall simply note them, omitting altogether the proofs. Other facts are rather deep and demand, for a rigorous proof, a certain amount of tedious and obscuring detail. For these latter facts we shall attempt to supply enough of a sketch so that the interested reader may see what sort of detail could be filled in to give a complete proof. Our intent here is very much like that of other authors in the field of recursive function theory who use "Church's Thesis" throughout their proofs, in order that the reader may follow the main ideas of the proofs more easily.

Lemma 2.3. For all $\alpha, \beta$ :

(i) If $S \in A_{\alpha}$ and $T$ is arithmetical in $S$, then $T \in A_{\alpha}$.

(ii) If $\alpha<\beta$ then $A_{\alpha} \subset A_{\beta}$.

Definition 2.4. Suppose $A \subset N$ is such that

$$
(\exists n)(x)[x \in A \Rightarrow \operatorname{seq}(x) \wedge \operatorname{lh}(x)=n] ;
$$


then we say that $A$ is an $n$-ary relation-set. The $n$-ary relation on integers corresponding to an $n$-ary relation-set $A$ is

$$
\left\{\left(x_{1}, \ldots, x_{n}\right) \mid\left\langle x_{1}, \ldots, x_{n}\right\rangle=p_{0}^{x_{1}+1} \cdots p_{n-1}^{x_{n}+1} \in A\right\}
$$

(where $p_{i}=$ the $i$ th odd prime; $p_{0}=2$ ).

If $A$ is an $(n+1)$-ary relation-set such that

$$
\left(x_{1}, \ldots, x_{n}\right)\left(\exists ! x_{n+1}\right)\left[\left\langle x_{1}, \ldots, x_{n}, x_{n+1}\right\rangle \in A\right]
$$

then we call $A$ an $n$-place function-set.

If $A$ is a 2-ary relation-set whose corresponding 2-ary relation is a well-ordering, then we say that $A$ is a well-ordering, and by $\|A\|$ we mean the ordinal which is the order-type of the well-ordering corresponding to $A$. Similarly, we say $x \in$ Fld $(A)$ if $x$ is in the field of the relation corresponding to $A$, and if $A$ corresponds to a well-ordering and $x \in$ Fld $(A)$, then by $|x|_{A}$ we mean the ordinal which is the order type of the segment determined by $x$ in the well-ordering corresponding to $A$.

We will sometimes say that a relation-set (function-set) represents the corresponding relation (function).

Definition 2.5. Suppose $A$ is a 2-place function-set, representing a function $f$, $f: N \times N \rightarrow\{0,1\}$; then $S_{i}^{A}=\{x \mid f(i, x)=1\}$. Thus a 2-place function-set $A$ can be used to represent in a unique way a countable collection of sets $\left\{S_{i}^{A}\right\}_{i \in N}$.

Suppose $A$ is a 2-ary relation-set which represents a linear ordering, and $B$ is a 3-place function-set representing a function

$$
f: \text { Fld }(A) \times N \times N \rightarrow\{0,1\} ;
$$

then for $a \in$ Fld $(A)_{B} S_{i}^{a}=\{x \mid f(a, i, x)=1\}$. Such a set $B$ "represents" in a natural way a linearly ordered collection (indexed by elements of Fld $(A)$ ) of countable families $\left\{{ }_{B} S_{i}^{a}\right\}_{i \in N}$ of sets.

LEMmA 2.6. There is a relation $R(X, Y)$ hyperarithmetical in the sets $X$ and $Y$, such that for any 2-place function-set $A$, there is a unique 2-place function-set $B$ such that $R(A, B)$, and such that if $R(A, B)$ then the family of sets $\left\{S_{i}^{B}\right\}_{i \in N}$ is exactly the family of all those sets which can be defined over the family $\left\{S_{i}^{A}\right\}_{i \in N}$ in 2-N.T. with set constants from $\left\{S_{i}^{A}\right\}_{i \in N}$. In particular, for any 2-place function-set $A$, the set $B$ such that $R(A, B)$, and each of the sets $S_{i}^{B}, i \in N$, are hyperarithmetical in $A$.

Proof. Set quantifiers and set constants over $\left\{S_{i}^{A}\right\}_{i \in N}$ can be expressed as number quantifiers and number constants before a matrix arithmetical in $A$. The method is illustrated by the following example: The formula

$$
(\alpha)_{F}(\exists \beta)_{F}(\gamma)_{F}(\exists x) \quad\left(x \in \alpha \vee x \in \beta \vee\left(y \in \gamma \rightarrow x \in S_{17}^{A}\right)\right),
$$

where " $F$ " denotes the family $\left\{S_{i}^{A}\right\}_{i \in N}$, defines the same set of $y$ as the $A$-arithmetical formula

$$
(i)(\exists j)(k)(\exists x)(f(i, x)=1 \vee f(j, x)=1 \vee(f(k, y)=1 \rightarrow f(17, x)=1)),
$$


where $f(x, y)=z \equiv_{\mathrm{df}} 2^{x+1} 3^{y+1} 5^{z+1} \in A$. Thus the sets definable by formulas of 2-N.T. over $F=\left\{S_{i}^{A}\right\}_{i \in N}$ (possibly using the constants " $S_{1}^{A}$ ", " $S_{2}^{A}$ ", $\ldots$ for members of $F$ ) are uniformly recursive in the set of (Gödel numbers of) arithmetical truths about $A$. The lemma follows from the fact that the relation $\operatorname{Tr}(X, y) \equiv\{y$ is the Gödel number of a true sentence arithmetical in $X\}$ is hyperarithmetical in $X$.

LEMMA 2.7. There is a hyperarithmetical relation $\operatorname{CONSTR}(X, Y)$ such that if $A$ is a 2-ary relation-set representing a well-ordering, then there is a unique $B$ such that $\operatorname{CONSTR}(A, B)$ and $B$ represents a function $f:$ Fld $(A) \times N \times N \rightarrow\{0,1\}$ such that if $a \in$ Fld $(A)$ then $A_{\alpha}=\left\{{ }_{B} S_{i}^{a}\right\}_{i \in N}$, where $\alpha=|a|_{A}$. In particular $B$ and each of the sets ${ }_{B} S_{i}^{a}, a \in$ Fld $(A), i \in N$, are hyperarithmetical in $A$.

Proof. Let $R$ be the relation introduced in Lemma 2.6. We define $\operatorname{CONSTR}(X, Y)$ as follows:

$\operatorname{CONSTR}(X, Y)$ iff:

(i) $X$ is a 2-ary relation-set which represents a linear ordering with least element and

(ii) $Y$ represents a function $f$ : Fld $(X) \times N \times N \rightarrow\{0,1\}$ and

(iii) if $x_{0}$ is the $X$-least element of Fld $(X)$, then $R\left(\varnothing,\left\{{ }_{Y} S_{i}^{x_{0}}\right\}_{i \in N}\right)$, where $\phi$ denotes the constant 0 2-place function-set, and

(iv) if $x^{\prime} \in \mathrm{Fld}(X)$ is the $X$-successor of $x \in$ Fld $(X)$ then

$$
R\left(\left\{S_{Y} S_{i}^{x}\right\}_{i \in N},\left\{{ }_{Y} S_{i}^{x^{\prime}}\right\}_{i \in N}\right) \text { and }
$$

(v) if $x \in$ Fld $(X)$ is not the $X$-least element of Fld $(X)$, nor a successor in the relation $<_{X}$ corresponding to $X$, then

(a) ${ }_{Y} S_{i}^{x}=\varnothing$ if $i \neq\langle n, a\rangle$ for all $n \in N, a \in$ Fld $(X), a<_{X} x$ or

(b) ${ }_{Y} S_{\langle n, a\rangle}^{x}={ }_{Y} S_{n}^{a}$ if $n \in N, a \in$ Fld $(X)$ and $a<{ }_{X} x$.

It is easy to see from the fact that $R$ is hyperarithmetical, that $\operatorname{CONSTR}(X, Y)$ is also hyperarithmetical. Existence and uniqueness of $Y$ follow by induction on $\|X\|$.

We close this section with a result of Kleene [K].

Lemma 2.8. $S \in A_{\omega_{1}} \Leftrightarrow S \in$ H.A.

Proof. $\Rightarrow$ : Suppose $S \in A_{\omega_{1}}$, then $S \in A_{\beta}$ for some $\beta<\omega_{1}$. Let $A$ be a recursive 2-ary relation-set such that $\|A\|=\beta+1$. Then by Lemma $2.7 S$ is hyperarithmetical in $A$, but, since $A$ is recursive, $S$ is hyperarithmetical.

$\Leftarrow$ : One can show by induction on $|a|_{0}$ that if $a \in O,|a|_{0}=\alpha$, then $H_{a} \in A_{\alpha+1}$. In fact, if " $\omega(a)$ " is a notation in $O$ for $\omega(1+\alpha)$, then $H_{\omega(a)} \in A_{\alpha+1}$, and so does $H_{\omega(a)+{ }_{0} n_{0}}$, for all $n$. Namely, suppose this holds for $\beta<\alpha<\omega_{1}$, and consider $A_{\alpha+1}$. By the induction hypothesis, and the fact that $A_{\beta+1} \subset A_{\alpha}$, the sets $H_{b}$ for $b<_{0} \omega(a)$ all belong to $A_{\alpha}$. Let $\omega(a)=3 \cdot 5^{u}$. Then $H_{\omega(a)}=H_{3} \cdot 5^{u}$ can be defined over $A_{\alpha}$ thus:

$$
\begin{aligned}
x \in H_{3} \cdot 5^{u} & \Leftrightarrow(x)_{0} \in H_{u_{(x)_{1}}} \\
& \Leftrightarrow(\exists \beta)_{A_{\alpha}}\left((x)_{0} \in \beta \& A\left(u_{(x)_{1}}, \beta\right)\right),
\end{aligned}
$$


where $A(e, \beta)$ is the arithmetical predicate which (for $e \in 0$ ) is satisfied only by the set $H_{e}$. Thus $H_{\omega(a)} \in A_{\alpha+1}$. Since the $A_{\alpha}$ are closed under "arithmetical in", it follows that $A_{\omega(a)+{ }_{0} n_{0}} \in A_{\alpha+1}$, for all $n$. [One can also show that $A_{\alpha+1}=$ the family of all sets arithmetical in $H_{\omega(a)}$ for any $a \in 0$ such that $|a|_{0}=\alpha$, but we do not need this here.]

3. The main theorem and main lemma. By Lemmas 1.8 and 2.8 we see that if $D$ is an a.d.h. on $\Sigma(\beta), \beta \geqq \omega_{1}$, then $R U\left\{D(\alpha) \mid \alpha<\omega_{1}\right\}=A_{\omega_{1}}=$ H.A. Thus the admissible degree-hierarchies and the ramified analytical hierarchy "pace" each other out to $\omega_{1}$. We shall show that they pace each other "all the way out."

Definition 3.1. Let $D$ be an a.d.h. on $\Sigma(\beta)$, then $D$ is complete if $D$ cannot be extended to an a.d.h. on $\Sigma(\alpha)$ for any $\alpha>\beta$.

TheOREM 3.2 (The MAIN TheOREM). (i) Let $D$ be an a.d.h. on $\Sigma(\beta)$, then $D$ is complete iff $\beta=\beta_{0}$.

(ii) There exists an a.d.h. on $\Sigma\left(\beta_{0}\right)$, and for any such a.d.h. $D, R U\left\{D(\alpha) \mid \alpha<\beta_{0}\right\}$ $=$ R.A.

The rest of this paper will be devoted to proving Theorem 3.2. The proof falls into two distinct halves. First we show that for various ordinals $\beta$ the equation $R U\{D(\alpha) \mid \alpha<\beta\}=A_{\beta}$ holds, and in particular that it holds for $\beta=\beta_{0}$. Second we show that any a.d.h. on $\Sigma\left(\beta_{0}\right)$ must be complete; in fact, we show that if $\mathfrak{M}$ is any countable $\omega$-model (in particular if $\mathfrak{M}=\mathscr{R} \cup\left\{D(\alpha) \mid \alpha<\beta_{0}\right\}=$ R.A.), then not only is there no n-least u.u.b. to $\mathfrak{M}$, but there is not even a u.u.b. to $\mathfrak{M}$ which is arithmetical in every such u.u.b. In the rest of this section we prove some preliminary results and state the Main Lemma essential to the proof of the first half of Theorem 3.2 .

Definition 3.3. Let $S$ be any set of integers. We define $O^{(i), s}, \omega_{1}^{(i), s}$ as follows:

$$
\begin{array}{ll}
O^{(0), S}=O^{S}, & \omega_{1}^{(0), S}=\omega_{1}^{S}, \\
O^{(n+1), S}=O^{O(n), S}, & \omega_{1}^{(n+1), S}=\omega_{1}^{O(n), S} .
\end{array}
$$

Thus $\omega_{1}^{(i), s}=1 . u . b . ~\left\{|a|_{O^{(i), s}} \mid a \in O^{(i), S}\right\}$. Let $\omega_{1}^{\infty, S}=1$. u.b. $\left\{\omega_{1}^{(i), s} \mid i \in N\right\}$. For $S$ $\in \operatorname{deg}(\varnothing)$, we write $\omega_{1}^{(i)}, O^{(i)}, \omega_{1}^{\infty}$ for $\omega_{1}^{(i), s}, O^{(i) s}, \omega_{1}^{\infty, s}$ respectively. By convention we set $O^{(-1), S}=S$.

THEOREM 3.4. Let $S$ be any set. Then there is an S-a.d.h. on $\Sigma\left(\omega_{1}^{\infty, S}\right)$ and for any such $S$-a.d.h. $D$ on $\Sigma\left(\omega_{1}^{\infty, S}\right)$ :

(i) $R U\left\{D(\beta) \mid \beta<\omega_{1}^{(n), s}\right\}=$ H.A. ${ }^{(n-1), s}$

(ii) $O^{(n), s}$ is a 2-least u.u.b. to $R U\left\{D(\beta) \mid \beta<\omega_{1}^{(n), s}\right\}$.

Proof. By Spector's hyperarithmetical quantifier theorem $O^{(n), s}$ is definable in the form

$$
x \in O^{(n), S} \equiv(\exists \alpha)_{\mathrm{H} . \mathrm{A} . O^{(n-1), S}(z) R(\alpha, z, x)}
$$

with $R$ recursive in $O^{(n-1), S}$. But this means that $O^{(n), S}$ is definable with two number 
quantifiers over any u.u.b. to H.A..$^{(n-1), s}$. Thus it is recursive in the second jump of any such u.u.b. Since $O^{(n), S}$ is certainly a u.u.b. to H.A. ${ }^{(n-1), S}$, it is a 2-least u.u.b. to H.A. ${ }^{(n-1), s}$. By Lemma 1.9 we have that

$$
R U\left\{D(\alpha) \mid \alpha<\omega_{1}^{(0), S}\right\}=\text { H.A. }{ }^{(-1), s},
$$

and the theorem now follows by induction on $n$, using Lemma 1.6 and the fact that if $D_{0} \subset D_{1} \subset \ldots \subset D_{i} \subset \ldots$ is a nested sequence of $S$-a.d.h.'s on $\Sigma\left(\omega_{1}^{(i), S}\right)$, respectively, then $D^{*}=\bigcup_{i \in N} D_{i}$ is an $S$-a.d.h. on $\Sigma\left(\omega_{1}^{\infty}, S\right)$.

THEOREM 3.5. (i) For all $n, A_{\omega_{1}^{(n)}}^{(n)}$ H.A. ${ }^{o(n-1)}$

(ii) $A_{\omega_{1}^{\infty}}$ is closed under the hyperjump operator $S \rightarrow O^{S}$, and $\omega_{1}^{\infty}$ is the smallest ordinal $\alpha$ such that $A_{\alpha}$ is closed under hyperjump.

Proof. (ii) is immediate from (i). We prove (i) by induction on $n$ :

(a) If $n=0$, this is exactly Lemma 2.8 .

(b) Suppose the result holds for all $m<n$; then $A_{\omega_{1}^{(n-1)}}^{(2)}$ H.A. ${ }^{\text {O(n-2) }}$. By the hyperarithmetical quantifier theorem we see that $O^{(n-1)} \in A_{\omega_{1}^{(n-1)}+1}$. By induction on $|a|_{o^{(n)}}$ we can see that for all $a \in O^{(n)}$,

$$
H_{a}^{O(n-1)} \in A_{\omega_{1}^{(n-1)}+|a| O(n)+1}
$$

and since $\omega_{1}^{(n-1)}+|a|_{o^{(n)}}+1<\omega_{1}^{(n)}$ for all $a \in O^{(n)}$, we have $H_{a}^{O^{(n-1)}} \in A_{\omega_{1}}^{(n)}$ for all $a \in O^{(n)}$. Thus, since $A_{\omega_{1}^{(n)}}^{(1)}$ closed under "recursive in", we have H.A. ${ }^{(n-1)}$ $\subset A_{\omega_{1}^{(n)}}$.

Conversely, for each $\alpha<\omega_{1}^{(n)}$, there is a relation-set $A$ recursive in $O^{(n-1)}$ such that $A$ represents a well-ordering of order-type $\alpha$, and by Lemma 2.7 each element of $A_{\alpha}$ is hyperarithmetical in $A$, and hence in $O^{(n-1)}$. Thus for all $\alpha<\omega_{1}^{(n)}, A_{\alpha} \subset$ H.A. ${ }^{(n-1)}$ so

$$
A_{\omega_{1}^{(n)}}^{(n)} \bigcup_{\alpha<\omega_{1}^{(n)}} A_{\alpha} \subset \text { H.A. } .^{(n-1)} .
$$

Thus $A_{\omega_{1}^{(n)}}^{(n)}$ H.A. ${ }^{(n-1)}$, and the induction step is completed, and the lemma is proved.

From Theorems 3.4 and 3.5 we obtain the following important corollary:

COROLlary 3.6. Let $D$ be any a.d.h. on $\Sigma\left(\omega_{1}^{\infty}\right)$, then $R U\left\{D(\alpha) \mid \alpha<\omega_{1}^{\infty}\right\}=A_{\omega_{1}^{\infty}}$.

From the proof of Theorem 3.5 we obtain the following generalization (by relativizing to $S$ ):

COROLlaRY 3.7. Suppose that $\lambda<\beta_{0}$ and there is a set $S \in A_{\lambda+1}$ such that $\omega_{1}^{S}>\lambda$, then:

(i) For all $n, A_{\omega_{1}^{(n), s}}^{(n)}$ H.A. ${ }^{(n-1), s}$

(ii) $\omega_{1}^{\infty}, s$ is the first ordinal $\alpha>\lambda$ such that $A_{\alpha}$ is closed under hyperjump.

REMARK. We see from Corollary 3.7 that what Theorems 3.4 and 3.5 depend on is the fact that there is a set $S_{n} \in A_{\omega_{1}^{(n)}+1}$ such that:

(i) $S_{n}$ is an $m$-least u.u.b. to $A_{\omega_{1}^{(n)}}$ for some $m$.

(ii) $\omega_{1}^{S_{n}}>\omega_{1}^{(n)}$. 
Suppose a similar thing happened at $A_{\omega_{1}^{\infty}}$, i.e., suppose there were a set $S \in A_{\omega_{1}^{\infty}+1}$ such that $S$ were an n-least u.u.b. to $A_{\omega_{1}^{\infty}}$ and $\omega_{1}^{S}>\omega_{1}^{\infty}$; then by Corollary 3.7 we would have for all $n, A_{\omega_{1}^{(n), s}}=$ H.A. ${ }^{(n-1), s}$, and by Theorem 3.4 and Lemma 1.6 we could construct an a.d.h. $D$ on $\Sigma\left(\omega_{1}^{\infty, S}\right)$ such that

$$
R U\left\{D(\alpha) \mid \alpha<\omega_{1}^{\infty, s}\right\}=A_{\omega_{1}^{\infty}, s}
$$

Thus we would have extended Theorems 3.4 and 3.5 to the ordinal $\omega_{1}^{\infty}, s$. Again suppose that there were a set $S^{\prime} \in A_{\omega_{1}^{\infty}{ }_{+}{ }_{+1}}$, such that $S^{\prime}$ were an n-least u.u.b. to $A_{\omega_{1}^{\infty}, S}$ and $\omega_{1}^{S^{\prime}}>\omega_{1}^{\infty, S}$; it would then be possible to extend the result to $\omega_{1}^{\infty, S^{\prime}}$. What we wish to show is that such continuation is always possible.

Definition 3.8. HYP $(\alpha)$ (" $\alpha$ is HYP") iff $A_{\alpha}$ is closed under hyperjump.

Definition 3.9. If $\alpha<\beta_{0}$, then $\alpha$ is attainable iff there is a set $A \in A_{\alpha+1}$ such that $A$ represents a well-ordering of order-type $\alpha$.

[In several abstracts, one of the authors defined " $\alpha$ is attainable" to mean: There is a set $A \in A_{\alpha+1}$ such that $A$ represents a well-ordering of order-type $\alpha$ all of whose initial segments are in $A_{\alpha}$. We have deleted the italicized clause as being unnecessary for the proof. However all theorems in the present paper are easily proved with the stronger definition of "attainable".]

LEMma 3.10. Suppose that $\lambda<\beta_{0}$ is an attainable ordinal and that $S \in A_{\lambda+1}$ such that $\|S\|=\lambda$; then all ordinals $\alpha$ such that $\lambda \leqq \alpha<\omega_{1}^{\infty}, s$ are attainable.

Proof. Assume that the conditions of the lemma are satisfied, then $\omega_{1}^{S}>\lambda$, so for all $n, A_{\omega_{1}^{(n) S}}=$ H.A..$^{o(n-1), s}$; let $\omega_{1}^{(-1), S}=\lambda$, then it is easy to see that for all $n \geqq 0$, if $\omega_{1}^{(n-1), s} \leqq \alpha<\omega_{1}^{(n), S}$, then there is a relation-set of order-type $\alpha$ in $A_{\omega_{1}^{(n-1), s}+1}$. But this clearly means that all ordinals $\alpha, \lambda \leqq \alpha<\omega_{1}^{\infty}, s$, are attainable.

REMARK. Clearly all ordinals $\alpha<\omega_{1}^{\infty}$ are attainable.

[It is necessary to recall that there is a well-ordering of order-type $\omega_{1}$ (in fact a path through $O$ ) which is recursive in $O$. By relativization, there is a well-ordering of order-type $\omega_{1}^{(n+1)}$ which is recursive in $O^{(n)}$ for all $n$.]

We now state the main lemma upon which the first half of the Main Theorem depends.

Lemma 3.11 (The Main Lemma). Suppose HYP $(\alpha), \alpha<\beta_{0}$, and all ordinals $\gamma<\alpha$ are attainable. Then there is a set $S \in A_{\alpha+1}$ such that:

(i) $S$ is a u.u.b. for $A_{\alpha}$.

(ii) $\omega_{1}^{S}>\alpha$.

We will postpone a proof of the Main Lemma until we indicate how it is used to prove the first half of the Main Theorem.

4. Proof of the first half of the Main Theorem assuming the Main Lemma. In this section we will assume Lemma 3.11 and use it to prove half of our Main Theorem: 
THEOREM 4.1. There is an a.d.h. on $\Sigma\left(\beta_{0}\right)$ and if $D$ is any such a.d.h. then

$$
R U\left\{D(\alpha) \mid \alpha<\beta_{0}\right\}=R . A .
$$

In order to prove Theorem 4.1 we first need a number of preliminary lemmas.

LEMMA 4.2. Let $\omega_{1}^{\infty} \leqq \alpha \leqq \beta_{0}$, then either

(a) $\mathrm{HYP}(\alpha)$ or

(b) There is a greatest HYP ordinal $\beta$ such that $\beta<\alpha$.

Proof. If $\alpha=\omega_{1}^{\infty}$ then (a) holds. Otherwise let $T_{\alpha}=\{\beta \mid \operatorname{HYP}(\beta) \wedge \beta<\alpha\}$. Let $\gamma=$ l.u.b. $T_{\alpha}$. Then obviously $A_{\gamma}=\bigcup_{\beta \in T_{\alpha}} A_{\beta}$. But, since a union of sets closed under hyperjump is also closed under hyperjump, we have HYP $(\gamma)$. Since $\gamma \leqq \alpha$, the lemma follows.

LEMMA 4.3. Every ordinal $\alpha<\beta_{0}$ is attainable, and all HYP ordinals are limit ordinals.

Proof. We will show by induction on $\alpha$ that

$$
\alpha<\beta_{0} \rightarrow(\alpha \text { attainable } \wedge(\operatorname{HYP}(\alpha) \rightarrow \operatorname{Lim} \alpha)) .
$$

If $\alpha<\omega_{1}^{\infty}$ then we know that the lemma holds. Now suppose that $\omega_{1}^{\infty} \leqq \beta<\beta_{0}$ and the result holds for all ordinals $\alpha<\beta$. One of the two following cases obtains:

CASE ONE. Suppose $\beta$ is HYP. Then by the Main Lemma there is a set $S \in A_{\beta+1}$ such that $\omega_{1}^{S}>\beta$. Hence some relation-set in $A_{\beta+1}$ must represent a relation of order-type $\beta$. Therefore $\beta$ is attainable. Also by Lemma 4.2 either $\beta$ is the limit of an increasing sequence of HYP ordinals in which case $\operatorname{Lim}(\beta)$, or there is a greatest HYP ordinal, say $\gamma$, less than $\beta$. In this latter case we can select a set $S \in A_{\gamma+1}$ such that $\omega_{1}^{S}>\gamma$. Hence by Corollary 3.7, $\omega_{1}^{\infty, S}$ is the least HYP ordinal greater than $\gamma$. Therefore $\beta=\omega_{1}^{\infty}, s$ and again $\operatorname{Lim}(\beta)$.

CASE Two. Suppose $\beta$ is not HYP and let $\alpha_{0}$ be the largest HYP ordinal less than $\beta$. Let $S \in A_{\alpha_{0}+1}$ such that $\|S\|=\alpha_{0}$; then $\omega_{1}^{S}>\alpha_{0}$, and by Corollary $3.7 \omega_{1}^{\infty}, S$ is the least HYP ordinal greater than $\alpha_{0}$; so $\alpha_{0}<\beta<\omega_{1}^{\infty}$,s. But then by Lemma $3.10 \beta$ must be attainable.

This completes the inductive proof.

LEMMA 4.4. If HYP $(\lambda)$ then there is an a.d.h. $D$ on $\Sigma(\lambda)$ and $R U\{D(\alpha) \mid \alpha<\lambda\}$ $=A_{\lambda}$.

Proof. By induction on $\lambda$. The first ordinal to consider is $\omega_{1}^{\infty}$, and the result for $\lambda=\omega_{1}^{\infty}$ is exactly Corollary 3.6.

Suppose now that $\lambda>\omega_{1}^{\infty}$ and that the result holds for all ordinals $\alpha<\lambda$. If $\lambda$ is not HYP then we are done. Suppose $\lambda$ is HYP, then there are two cases to consider:

CASE ONE. Suppose there exists a largest HYP ordinal $\alpha_{0}<\lambda$. In this case there is an a.d.h. $D$ on $\Sigma\left(\alpha_{0}\right)$, and $R U\left\{D(\gamma) \mid \gamma<\alpha_{0}\right\}=A_{\alpha_{0}}$. By the Main Lemma together with Lemma 4.3 there is a set $S_{0} \in A_{\alpha_{0}+1}$ such that

(i) $\omega_{10}^{S}>\alpha_{0}$

(ii) $S_{0}$ is a u.u.b. to $A_{\alpha_{0}}$. 
We first observe that the fact that $S_{0}$ has a definition in 2-N.T. over $A_{\alpha_{0}}$ (perhaps with constant terms) means that for some $n, S_{0}$ is an n-least u.u.b. to $A_{\alpha_{0}}$. The argument for this is essentially the one indicated in the proof of Theorem 3.4 for the fact that $O^{(n)}$ is a 2-least u.u.b. to H.A. ${ }^{O^{(n-1)}}$. By Corollary 3.7 we know that $\lambda=\omega_{1}^{\infty}, s_{0}$. By Theorem 3.4, there is an $S_{0}$-a.d.h. $D^{\prime}$ on $\Sigma\left(\omega_{1}^{\infty}, s_{0}\right)$. Let $D^{*}$ be defined as follows:

if $\alpha<\alpha_{0}, D^{*}(\alpha)=D(\alpha)$,

if $\beta<\omega_{1}^{\infty}, s_{0}, D^{*}\left(\alpha_{0}+\beta\right)=D^{\prime}(\beta)$.

Then by Lemma 1.6, $D^{*}$ is an a.d.h. on $\Sigma\left(\alpha_{0}+\lambda\right)$. But since $\alpha_{0}<\omega_{1}^{(0), s_{0}}$, we have $\alpha_{0}+\lambda=\lambda$; so $D^{*}$ is an a.d.h. on $\Sigma(\lambda)$. By Theorem 3.4,

$$
R U\left\{D^{\prime}(\alpha) \mid \alpha<\omega_{1}^{(n), s_{0}}\right\}=\text { H.A. } .^{(n-1), s_{0}},
$$

but, since for all $n, \alpha_{0}+\omega_{1}^{(n), s_{0}}=\omega_{1}^{(n), s_{0}}$, we have

$$
R U\left\{D^{*}(\alpha) \mid \alpha<\omega_{1}^{(n), s_{0}}\right\}=\text { H.A. }{ }^{(n-1), s_{0}} .
$$

By Corollary 3.7 we also have $A_{\omega_{1}^{(n), s_{0}}}=$ H.A..$^{(n-1), s_{0}}$. Thus

$$
A_{\lambda}=A_{\omega_{1}^{\infty}, s_{0}}=\bigcup_{n \in N} A_{\omega_{1}^{(n), s_{0}}}=R U\left\{D^{*}(\alpha) \mid \alpha<\lambda\right\}
$$

so $D^{*}$ has the required properties.

CASE Two. Suppose there is no largest HYP ordinal less than $\lambda$. Then $\lambda$ is the limit of an increasing sequence $\left\{\alpha_{n}\right\}$ of HYP ordinals. Now, using the inductive hypothesis applied to the $\alpha_{n}$ 's and Lemma 1.7, we can string together a.d.h.'s on the $\alpha_{n}$ 's to get an a.d.h. on $\lambda$ with the desired property.

LEMMA 4.5. HYP $\left(\beta_{0}\right)$.

Proof. Suppose $\beta_{0}$ were not HYP, and let $\gamma_{0}$ be the largest HYP ordinal less than $\beta_{0}$. Let $S \in A_{\gamma_{0}+1}$ be such that $\omega_{1}^{S}>\gamma_{0}$ (Lemma 3.11). Then by Corollary 3.7 we have for all $n$,

$$
A_{\omega_{1}^{(n) . S}}=\text { H.A. }{ }^{\mathrm{O}^{(n-1), s}} \neq A_{\omega_{1}^{(n+1), s}}=\mathrm{H} \cdot \mathrm{A} .^{\mathrm{o(n),s}} ;
$$

so $\omega_{1}^{\infty}, s \leqq \beta_{0}$, and $\omega_{1}^{\infty, s}$ is HYP, so $\omega_{1}^{\infty}, s<\beta_{0}$; but this contradicts the choice of $\gamma_{0}$ as the largest HYP ordinal less than $\beta_{0}$. Thus $\beta_{0}$ must be HYP.

Theorem 4.1 now follows immediately from Lemmas 4.4 and 4.5.

We now turn our attention to proving the Main Lemma.

\section{Proof of the Main Lemma.}

DEFINITION 5.1. We will abbreviate by W.O. $(X)$ the formula of 2-N.T. with one free set variable as follows:

$W . O .(X) \equiv[[X$ is a 2-ary relation-set $] \wedge[X$ represents a linear ordering $] \wedge(S)$ [ $[S$ is a 1-place function set representing a function $f$ such that Range $(f) \subset$ Fld $(X)$ $\wedge(n)[\langle f(n+1), f(n)\rangle \in X]] \Rightarrow(\exists m)(z)[z \geqq m \Rightarrow f(z)=f(m)]]]$, where the English expressions between braces $([]$,$) are understood to be written as arithmetical$ expressions in the appropriate variables. 
If the set quantifier in W.O. $(X)$ is understood to range over $2^{N}$ then W.O. $(X)$ simply says that $X$ is a well-ordering. We need to know that this is true under certain other conditions.

Notational Remark 5.2. If $\mathfrak{R} \subset 2^{N}, \varphi\left(X_{1}, \ldots, X_{n}\right)$ is a formula of 2-N.T. with free set variables $X_{1}, X_{2}, \ldots, X_{n}$, and $A_{1}, \ldots, A_{n} \in \mathfrak{\Re}$, we will write " $\mathfrak{\AA}(\mathrm{SAT})$ $\varphi\left(A_{1}, \ldots, A_{n}\right)$ " when we mean that $\varphi$ holds in $\Re$ with respect to the sequence $A_{1}, \ldots, A_{n}$.

Lemma 5.3. Suppose $\left(X_{1}, \ldots, X_{n}, x_{1}, \ldots, x_{m}\right)$ is a relation $\Pi_{1}^{1}$ in $X_{1}, \ldots, X_{n}$. If $\Re \subset 2^{N}$ has the following properties:

(i) if $X, Y \in \Re$, then $X \times Y=\{\langle i, j\rangle \mid i \in X \& j \in Y\} \in \Re$,

(ii) $\Re$ is closed under hyperjump and "recursive in": then, for all $A_{1}, \ldots, A_{n}$ $\in \Re, i_{1}, \ldots, i_{m} \in N$,

$$
R\left(A_{1}, \ldots, A_{n}, i_{1}, \ldots, i_{m}\right) \equiv \AA(S A T) R\left(A_{1}, \ldots, A_{n}, i_{1}, \ldots, i_{m}\right) .
$$

Proof. Let $A_{1}, \ldots, A_{n} \in \Re$ and let $A=A_{1} \times A_{2} \times \cdots \times A_{n}$. It is obvious that $R\left(A_{1}, \ldots, A_{n}, x_{1}, \ldots, x_{m}\right)$ is expressible in the form $S\left(A, x_{1}, \ldots, x_{m}\right)$ where $S$ is $\Pi_{1}^{1}$ in $A$. But by (i), $A \in \Re$ and by (ii), $O^{A} \in \Re$. Again, by (ii) and the well-known fact that $O^{A}$ is a complete $\Pi_{1}^{1}$-in- $A$ predicate, it follows that the extension of $S\left(A, x_{1}, \ldots, x_{m}\right)$ and hence of $R\left(A_{1}, \ldots, A_{n}, x_{1}, \ldots, x_{m}\right)$ is a member of $\AA$. Hence by Kleene [K', p. 324, $\$ 5.5$, Remark 5], it follows that

$$
R\left(A_{1}, \ldots, A_{n}, i_{1}, \ldots, i_{m}\right) \equiv \mathfrak{\Re}(\mathrm{SAT}) R\left(A_{1}, \ldots, A_{n}, i_{1}, \ldots, i_{m}\right) .
$$

REMARK 5.4. In order to apply Remark 5.2 to an analytic relation $R\left(X_{1}, \ldots, X_{n}\right.$, $\left.x_{1}, \ldots, x_{m}\right)$ we certainly must think of the relation as written in some definite way as a formula of 2-N.T. In Lemma 5.3, for example, $R\left(X_{1}, \ldots, X_{n}, x_{1}, \ldots, x_{m}\right)$ is thought of as being a $\Pi_{1}^{1}$ formula of 2-N.T. Also in reference to Lemma 5.3 it is easy to see that if HYP $(\alpha)$ then $\mathfrak{\Re}=A_{\alpha}$ does satisfy properties (i) and (ii) of the lemma.

Definition 5.5. Let $\sigma$ be the following sentence of 2-N.T.:

$$
\begin{aligned}
(X)(\exists Y)(\exists Z)\left[W . O .(Y) \wedge \operatorname{CONSTR}(Y, Z) \wedge(\exists a)(\exists i)\left[a \in \text { Fld }(Y) \wedge X={ }_{z} S_{i}^{a}\right]\right] \\
\wedge(X)(Y)(\exists Z)[Z=X \times Y] \wedge(e)(X)(\exists Y)(x)\left[x \in Y \Leftrightarrow(\exists z) T^{x}(e, x, z)\right]
\end{aligned}
$$

where $T^{x}(e, x, z)$ is the Kleene $T$-predicate relativized to $X$.

LEMMA 5.6. (i) If HYP $(\alpha)$ and $(\beta)[\beta<\alpha \rightarrow \beta$ is attainable $]$, then $A_{\alpha}(\mathrm{SAT}) \sigma$.

(ii) If $\Re \subset 2^{N}$ is closed under hyperjump and $\Re(\mathrm{SAT}) \sigma$, then $(\exists \beta)\left[\Re=A_{\beta}\right]$.

Proof. (i) Suppose HYP ( $\alpha$ ) and all ordinals $\beta<\alpha$ are attainable. The second and third clauses of $\sigma$ simply assert closure under "cross-product" and "recursively enumerable in" and it is immediate that $A_{\alpha}$ satisfies these clauses.

In order to see that $A_{\alpha}$ satisfies the first clause, suppose that $S \in A_{\alpha}$; since all HYP ordinals are limit ordinals, $S \in A_{\beta}$ for some $\beta<\alpha$. Since all ordinals $\gamma<\alpha$ are 
attainable there must be a relation-set $A \in A_{\beta+1}$ such that $\|A\|=\beta$. Let $B$ be the unique set such that $\operatorname{CONSTR}(A, B)$, then $B$ is hyperarithmetical (hence, $\left.\Pi_{1}^{1}\right)$ in $A$, so $B \in A_{\alpha}$. By 5.3 and 5.4 we see that

$$
A_{\alpha}(\operatorname{SAT})\left[W . O .(A) \wedge \operatorname{CONSTR}(A, B) \wedge(\exists a)(\exists i)\left[a \in \text { Fld }(A) \wedge S={ }_{B} S_{i}^{a}\right]\right] .
$$

But since $S$ was an arbitrary set in $A_{\alpha}$ we see that $A_{\alpha}$ must satisfy the first clause of $\sigma$, thus $A_{\alpha}(\mathrm{SAT}) \sigma$.

(ii) Suppose $\mathfrak{\Re}(\mathrm{SAT}) \sigma$ and $\mathfrak{\AA}$ is closed under hyperjump. Then the second and third clauses of $\sigma$ require that $\AA$ be closed under "cross-product" and "recursively enumerable in" so that $\AA$ meets the conditions of Lemma 5.3. Let $\beta$ be the least ordinal such that if $A \in \mathfrak{\Omega}$ represents a well-ordering, then $\|A\| \leqq \beta$. Then it is easy to check that $\AA=A_{\beta}$.

We now wish to show that a form of the axiom of choice holds in $A_{\alpha}$ whenever HYP $(\alpha)$ and all ordinals $\beta<\alpha$ are attainable.

THEOREM 5.7. There is a formula BEFORE $(X, Y)$, of 2-N.T. such that whenever $\mathrm{HYP}(\alpha)$ and all ordinals $\beta<\alpha$ are attainable, then the relation on sets $X R Y \equiv$ $A_{\alpha}(\mathrm{SAT}) \operatorname{BEFORE}(X, Y)$ is a well-ordering of $A_{\alpha}$.

Our proof of Theorem 5.7 will be more complicated than might at first seem necessary. The natural first move in proving the theorem would be to try to define $B E F O R E$ directly from the relations $W . O$. and CONSTR as follows:

$B E F O R E(X, Y)$ iff $(\exists Z)(\exists W)$ such that

(i) W.O. (Z) $\wedge \operatorname{CONSTR}(Z, W)$ and

(ii) $(\exists a)(\exists b)(\exists i)(\exists j)\left[X={ }_{w} S_{i}^{a} \wedge Y={ }_{w} S_{j}^{b}\right]$ and

(iii) if $R$ is the relation

$$
\left\langle x_{1}, x_{2}\right\rangle R\left\langle y_{1}, y_{2}\right\rangle \equiv x_{1}, y_{1} \in \text { Fld }(Z) \wedge\left[\left\langle x_{1}, y_{1}\right\rangle \in Z \vee\left(\left(x_{1}=y_{1}\right) \wedge\left(x_{2}<y_{2}\right)\right)\right]
$$

then the $R$-least number $\langle a, i\rangle$ such that $X={ }_{W} S_{i}^{a} R$-precedes the $R$-least number $\langle b, j\rangle$ such that $Y={ }_{W} S_{j}^{b}$.

Where this definition fails is that the relation depends on the choice of the set $Z$ in the following way: We know from Lemma 2.7 that if $Z_{1}$ and $Z_{2}$ represent wellorderings and Constr $\left(Z_{1}, W_{1}\right)$, Constr $\left(Z_{2}, W_{2}\right)$, then $\left\{w_{1} S_{i}^{a}\right\}_{i \in N}=\left\{W_{2} S_{i}^{b}\right\}_{i \in N}$ whenever $|a|_{z_{1}}=|b|_{z_{2}}$. But we have no guarantee that under these conditions we have the identity ${ }_{w_{1}} S_{i}^{a}={ }_{w_{2}} S_{i}^{b}$ for all $i \in N$. But the relation defined above depends on the particular order of the sequence $\left\{{ }_{w} S_{i}^{a}\right\}_{i \in N}$. Thus we are forced to find a relation which does not depend on the order of the sets ${ }_{w} S_{i}^{a}$ but only on membership in the family of sets $\left\{w S_{i}^{a}\right\}$.

LEMMA 5.8. For any ordinal $\alpha$ and any set $B \in A_{\alpha}, B$ can be defined by some w.f.f. of 2-N.T. without constant symbols, but with each of its set quantifiers restricted to some $A_{\beta}, \beta<\alpha$.

Proof. This follows easily by induction on $\alpha$. 
Definition 5.9. Let $A$ be a relation-set which represents a linear ordering. By $\mathfrak{L}_{A}$ we will mean the formal language which is just the language of 2-N.T. except that the set quantifiers all have the following special form: $\left(X_{i}\right)_{a}$ or $\left(\exists X_{i}\right)_{a}$ where $a \in$ Fld $(A)$. By $\mathfrak{L}_{A}^{\prime}$ we mean the subset of $\mathfrak{L}_{A}$ consisting of all those formulas which are in prenex normal form and have exactly one free number variable, and no free set variables.

Definition 5.10. Let $A$ and $B$ represent well-orderings and $\varphi \in \mathfrak{R}_{A}^{\prime}, \varphi^{\prime} \in \mathfrak{L}_{B}^{\prime}$. Then we say $\varphi \cong \varphi^{\prime}$ ( $\varphi$ is equivalent to $\varphi^{\prime}$ ) iff $\varphi$ and $\varphi^{\prime}$ are exactly alike except that wherever the quantifier $\left(\exists X_{i}\right)_{a}$ or $\left(X_{j}\right)_{b}$ occurs in $\varphi$, the quantifier $\left(\exists X_{i}\right)_{a^{\prime}}$ or $\left(X_{j}\right)_{b^{\prime}}$ respectively occurs in $\varphi^{\prime}$ where $|a|_{A}=\left|a^{\prime}\right|_{B},|b|_{A}=\left|b^{\prime}\right|_{B}$.

If we chose any natural Gödel numbering on the formulas of 2-N.T. which have integer subscripts on their set quantifiers, then it is obvious that the linear order represented by $A$ induces a natural linear order on (Gödel numbers of ) $\mathfrak{L}_{A}^{\prime}$ which depends only on the order-type of $A$, in the sense of the following lemma whose proof we leave to the reader:

LEMMA 5.11. There is an arithmetical formula SHORTER $(X, x, y)$ such that if $A$ represents a well-ordering, then $\operatorname{SHORTER}(A, x, y)$ defines a well-ordering on Gödel numbers of formulas in $\Omega_{A}^{\prime}$, and such that if $A_{1}$ and $A_{2}$ both represent wellorderings $\varphi_{1} \in{\mathfrak{\mathfrak { L } ^ { \prime }}}_{A_{1}}^{\prime}, \varphi_{2} \in \mathfrak{L}_{A_{2}}^{\prime}$ with Gödel numbers $q_{1}, q_{2}$ respectively and $\varphi_{1} \cong \varphi_{2}$ then the ordinal associated with $q_{1}$ in the relation $\operatorname{SHORTER}\left(A_{1}, x, y\right)$ is the same as the ordinal associated with $q_{2}$ in the relation $\operatorname{SHORTER}\left(A_{2}, x, y\right)$.

Definition 5.12. Let $A$ represent a linear ordering and let $B$ represent a function $f:$ Fld $(A) \times N \times N \rightarrow\{0,1\}$. We define a satisfaction relation (SAT) ${ }^{\prime}$ as follows:

$B(\mathrm{SAT})^{\prime} z$ iff $z=\left\langle n_{1}, n_{2}\right\rangle$ where $n_{1}$ is the Gödel number of a formula $\varphi(x)$ in $\mathfrak{L}_{A}^{\prime}$ and $\varphi\left(n_{2}\right)$ holds when the quantifier $\left(X_{i}\right)_{a}$ or $\left(\exists X_{i}\right)_{a}$ in $\varphi$ is understood to range over the family of sets $\left\{{ }_{B} S_{j}^{a}\right\}_{j \in N}$.

LEMMA 5.13. The relation $X(\mathrm{SAT})^{\prime} x$ is hyperarithmetical in $X$, and hence is univariant, in the sense of Lemma 5.3, over any $\mathfrak{\AA} \subset 2^{N}$ which satisfies the conditions of Lemma 5.3.

Proof. This follows as in Lemma 2.6 from the fact that arithmetical truth in $X$ is hyperarithmetical in $X$.

The satisfaction relation (SAT) ${ }^{\prime}$ was so defined as to make the following lemma trivial:

LEMMA 5.14. Suppose $A_{1}$ and $A_{2}$ represent well-orderings, $\operatorname{CONSTR}\left(A_{1}, B_{1}\right)$, $\operatorname{CONSTR}\left(A_{2}, B_{2}\right), \varphi_{1} \in \mathfrak{R}_{A_{1}}^{\prime}$ and $\varphi_{2} \in \mathfrak{L}_{A_{2}}^{\prime}$ with Gödel numbers $q_{1}$ and $q_{2}$ respectively; and $\varphi_{1} \cong \varphi_{2}$, then for all $n$,

$$
B_{1}(\mathrm{SAT})^{\prime}\left\langle q_{1}, n\right\rangle \Leftrightarrow B_{2}(\mathrm{SAT})^{\prime}\left\langle q_{2}, n\right\rangle .
$$

Proof. This is immediate from the fact that if $\left|a_{1}\right|_{A_{1}}=\left|a_{2}\right|_{A_{2}}$, then $\left\{{ }_{B_{1}} S_{i}^{a} a_{1}\right\}=$ $\left\{{ }_{B_{2}} S_{i}^{a_{2}}\right\}$ and from the definition of $\cong$ and (SAT)'. 
We are now in a position to define the relation $B E F O R E$.

DEFINITION 5.15. Let $B E F O R E(X, Y)$ be the following formula of 2-N.T.:

$$
\begin{aligned}
(\exists Z)(\exists W)[W . O .(Z) & \wedge \operatorname{CONSTR}(Z, W) \wedge(\exists x, y, u, v)\left(X={ }_{w} S_{y}^{x} \wedge Y={ }_{w} S_{v}^{u}\right) \\
& \wedge(y)\left((u)\left(u \in Y \Leftrightarrow W(S A T)^{\prime}\langle y, u\rangle\right)\right. \\
& \left.\left.\rightarrow(\exists x)\left(\operatorname{SHORTER}(Z, x, y) \wedge(z)\left(z \in X \Leftrightarrow W(S A T)^{\prime}\langle x, z\rangle\right)\right)\right)\right] .
\end{aligned}
$$

It is obvious from Lemmas 5.3, 5.4 and the lemmas following Theorem 5.7 that $B E F O R E$ has the properties required by Theorem 5.7, and thus that Theorem 5.7 is proved.

We are now in a position to make use of the definable well-ordering $B E F O R E$ to define Skolem-functions for sentences over $A_{\alpha}$.

LEMMA 5.16. Suppose HYP $(\alpha)$ and all ordinals $\beta<\alpha$ are attainable. Let $\varphi(x)$ be any formula of 2-N.T. (perhaps with set constants from $A_{\alpha}$ ) which is in prenex normal form. Then Skolem-functions for the sentences $\varphi(n)$ over $A_{\alpha}$ are definable over $A_{\alpha}$ uniformly in $n \in N$, in the sense of the following example:

Suppose $\varphi(x) \equiv\left(X_{1}\right)\left(\exists X_{2}\right)\left(X_{3}\right)\left(\exists X_{4}\right) A\left(X_{1}, \ldots, X_{4}, x\right)$ where $A$ is arithmetical in $X_{1}, \ldots, X_{4}, x$ and perhaps some set constants $A_{1}, \ldots, A_{n}$ from $A_{\alpha}$. Then what the lemma requires is that there be formulas $\varphi_{1}\left(x, X_{1}, X_{2}\right), \varphi_{2}\left(x, X_{1}, X_{3}, X_{4}\right)$ of 2-N.T. perhaps involving the constants from $A_{\alpha}$ such that:

(i) $A_{\alpha}(\mathrm{SAT})(x)\left(X_{1}\right)\left(\exists ! X_{2}\right) \varphi_{1}\left(x, X_{1}, X_{2}\right)$, $A_{\alpha}(\operatorname{SAT})(x)\left(X_{1}\right)\left(X_{3}\right)\left(\exists ! X_{4}\right) \varphi_{2}\left(x, X_{1}, X_{3}, X_{4}\right)$,

i.e. over $A_{\alpha}, \varphi_{1}$ and $\varphi_{2}$ define functions $f_{1}$ and $f_{2}$ respectively, $f_{1}: N \times A_{\alpha} \rightarrow A_{\alpha}$, $f_{2}: N \times A_{\alpha} \times A_{\alpha} \rightarrow A_{\alpha}$.

(ii) for all $n \in N$,

(a) if $A_{\alpha}(\mathrm{SAT}) \varphi(n)$ then $\lambda X_{1}\left[f_{1}\left(n, X_{1}\right)\right]$ and $\lambda X_{1} X_{3}\left[f_{2}\left(n, X_{1}, X_{3}\right)\right]$ are Skolemfunctions for $\varphi(n)$ over $A_{\alpha}$.

(b) if $A_{\alpha}(\mathrm{SAT}) \neg \varphi(n)$ then for all $X_{1}, X_{3} \in A_{\alpha}, f_{1}\left(n, X_{1}\right)=f_{2}\left(n, X_{1}, X_{3}\right)=\varnothing$.

Proof. The construction, e.g., of $\varphi_{1}$ and $\varphi_{2}$ is immediate from the fact that we can use the predicate $B E F O R E$ to define a choice function over $A_{\alpha}$. We leave the details to the reader.

LEMMA 5.17. Suppose HYP ( $\alpha)$ and all ordinals $\beta<\alpha$ are attainable, and suppose that functions $f_{1}, \ldots, f_{n}, f_{i}: N \times A_{\alpha}^{m_{i}} \rightarrow A_{\alpha}$ or $f_{i}: A_{\alpha}^{m_{i}} \rightarrow A_{\alpha}$, for some $m_{i} \in N$, are definable over $A_{\alpha}$ in 2-N.T. (perhaps with set constants from $A_{\alpha}$ ). Let $A_{1}, \ldots, A_{m}$ be any finite number of sets in $A_{\alpha}$. Then there is a 2-place function-set $A \in A_{\alpha+1}$ such that the family of sets $\left\{S_{i}^{A}\right\}_{i \in N}$ is exactly the closure of $\left\{A_{1}, \ldots, A_{m}, \varnothing\right\}$ and $N$ under the functions $f_{1}, \ldots, f_{n}$.

Proof. Consider the process of taking the sets $A_{1}, \ldots, A_{m}, \varnothing$ and $N$ and closing them under the functions $f_{1}, \ldots, f_{n}$, which mimics the proof that the closure of a countable set under countably many functions is again countable. It is straightforward but intricate to show that this process is describable over $A_{\alpha}$ in 2-N.T. with 
constants for sets in $A_{\alpha}$, in such a way that the relation $R(i, x, y) \equiv$ [either $x$ is in the $i$ th set generated in this way and $y=1$ or $x$ is not in the $i$ th set generated in this way and $y=0]$ is definable over $A_{\alpha}$. We leave the details to the reader. The set $A \in A_{\alpha+1}$ corresponding to the definition of $R$ over $A_{\alpha}$ is the desired element of $A_{\alpha+1}$.

We are now prepared to prove:

Lemma 3.11 (MaIn Lemma). Suppose HYP $(\alpha)$ and all ordinals $\beta<\alpha$ are attainable and $\alpha<\beta_{0}$. Then there is a set $S \in A_{\alpha+1}$ such that (i) $S$ is a u.u.b. for $A_{\alpha}$, (ii) $\omega_{i}^{S}>\alpha$.

Proof. Suppose the conditions of the lemma obtain, then since $\alpha<\beta_{0}, A_{\alpha+1}-A_{\alpha}$ $\neq \varnothing$. Let $B$ be any element of $A_{\alpha+1}-A_{\alpha}$, and let $\varphi(X)$ be a formula of 2-N.T. (perhaps with set constants from $A_{\alpha}$ ) which is in prenex normal form and defines $B$ over $A_{\alpha}$.

Consider the following formulas of 2-N.T.:

$$
\begin{aligned}
& \varphi_{1}(x) \equiv \varphi(x) \wedge \sigma \wedge(X)(\exists Y)\left[Y=O^{X}\right], \\
& \varphi_{2}(x) \equiv \neg \varphi(x) \wedge \sigma \wedge(X)(\exists Y)\left[Y=O^{X}\right],
\end{aligned}
$$

where " $Y=O^{X}$ " is written as in Lemma 5.3, and $\sigma$ is the sentence defined in Definition 5.5. Let $A_{1}, \ldots, A_{m}$ be the sets in $A_{\alpha}$ mentioned by set constants in $\varphi$.

By Lemmas 5.16 and 5.17, there must be a set $S \in A_{\alpha+1}$ such that $S$ is a 2-place characteristic function-set and the family of sets $\left\{S_{i}^{S}\right\}_{i \in N}$ is exactly the closure of $\left\{A_{1}, \ldots, A_{m}, \varnothing\right\}$ and $N$ under Skolem-functions over $A_{\alpha}$ for the sentences $\varphi_{i}(n)$, $i=1,2$ (in the sense of Lemma 5.16). Let $\Re=\left\{S_{i}^{S}\right\}_{i \in N}$. We first observe that, since $A_{\alpha}(\mathrm{SAT}) \sigma$, it must be the case that $\mathfrak{\AA}(\mathrm{SAT}) \sigma$. Since $A_{\alpha}$ is closed under hyperjump, the Skolem-functions for the third clauses of $\varphi_{1}$ and $\varphi_{2}$ actually define the hyperjump operator, thus $\Re$ must be closed under hyperjump. Hence by Lemma 5.6, $\mathfrak{R}=A_{\lambda}$ for some $\lambda$. Since $\mathfrak{\Re} \subset A_{\alpha}$, it is clear that $\lambda \leqq \alpha$. But $\mathfrak{I}$ is closed under the Skolem-functions for all the sentences $\varphi(n)$ and $\neg \varphi(n)$, and $A_{1}, \ldots, A_{m} \in \Re$. Thus $\varphi(x)$ defines $B$ over $\mathfrak{R}=A_{\lambda}$; but $B \in A_{\alpha+1}-A_{\alpha}$, so it must be that $\lambda=\alpha$, i.e., the family $\left\{S_{i}^{S}\right\}_{i \in N}$ is exactly $A_{\alpha}$. Thus $S$ is surely a u.u.b. to $A_{\alpha}$.

It remains to show that $\omega_{1}^{S}>\alpha$. Consider the following relation $R(x, y)$ :

$R(x, y)$ iff

(i) $x=\left\langle(x)_{1},(x)_{2}\right\rangle \wedge y=\left\langle(y)_{1},(y)_{2}\right\rangle$ and

(ii) $(x)_{2} \in S_{(x)_{1}}^{S} \wedge(y)_{2} \in S_{(y)_{1}}^{S}$ and

(iii) $S_{(x)_{1}}^{S}$ and $S_{(y)_{1}}^{S}$ represent well-orderings and

(iv) either $(x)_{1}<(y)_{1}$, or $(x)_{1}=(y)_{1}$ and $(x)_{2}$ precedes $(y)_{2}$ in the well-ordering corresponding to $S_{(x)_{1}}^{S}=S_{(y)_{1}}^{S}$.

Clearly $R$ represents a well-ordering, and since all ordinals $\beta<\alpha$ are represented by some $S_{i}^{S}$, the order-type of $R$ is at least $\alpha$. By 5.3 and 5.4 we see that $S_{i}^{S}$ represents a well-ordering just in case it represents a linear ordering in which none of the sets $S_{j}^{S}$ represents an infinite descending chain; thus clause (iii) is arithmetical 
in $S$, as are all the other clauses. Hence there are certainly well-orderings arithmetical in $S$ which have order-type greater than $\alpha$. But any ordinal which is arithmetical, or even $\Sigma_{1}^{1}$ in $S$ is recursive in $S$, thus $\omega_{1}^{S}>\alpha$ and the lemma is proved.

6. Proof of the second half of the Main Theorem. The purpose of this section is to establish the second half of the Main Theorem:

THEOREM 6.1. Any a.d.h. on $\Sigma\left(\beta_{0}\right)$ is complete.

This result will be obtained as an immediate corollary to the following theorem:

THEOREM 6.2. Let $\mathfrak{M}$ be a countable w-model for analysis, then there is no u.u.b. for $\mathfrak{M}$ which is arithmetical in every u.u.b. to $\mathfrak{M}$. [N.B. Let $\mathfrak{A}$ be the universe of sets for $\mathfrak{M}$.]

The idea of the proof is to define forcing suitably so that for any such $\mathfrak{M}$ we can get two generic uniform upper-bounds for $\mathfrak{A}$. It will turn out that forcing is defined so that any set which is arithmetical in both u.u.b.'s will have to be first-order definable over $\mathfrak{M}$, and hence will be a member of $\mathfrak{A}$. Since no u.u.b. for $\mathfrak{A}$ can be itself a member of $\mathfrak{A}$, we obtain the desired result.

In order to carry out the proof, we shall concern ourselves with 2 nd-order relational structures of the form:

$$
\mathfrak{N}=\left\langle N ; \mathfrak{A} ;+; \times ; B_{1} ; B_{2} ; \ldots, n, \ldots ; \ldots, a, \ldots\right\rangle
$$

where

(i) $N=\{0,1,2, \ldots\}$,

(ii) $\mathfrak{A} \subset 2^{N}$,

(iii) + and $\times$ are the three place relations over $N$ which represent addition and multiplication respectively,

(iv) $\ldots, n, \ldots$ is the sequence $0,1,2,3, \ldots ; \ldots, a, \ldots$ is an enumeration of the elements of $\mathfrak{A}$,

(v) $B_{1}, B_{2} \subset N$, but need not be elements of $\mathfrak{A}$.

The point of (iv) is that we want the language suitable for the structure $\mathfrak{N}$ to have an individual constant for each nonnegative integer, and a predicate constant for each element of $\mathfrak{A}$.

DefinItion 6.3. For any countable set $\mathfrak{A} \subset 2^{N}$, we define a language $\mathfrak{Q}_{\mathfrak{A}}$ suitable for such a structure as follows:

(1) The predicate constants in $\mathfrak{Q}_{\mathfrak{A}}$ are:

(a) $+(,$, ) and $\times(,$,$) , both 3-ary.$

(b) A 1-ary predicate constant $a(\quad)$ for each element $a$ of $\mathfrak{A}$.

(c) Two distinguished 1-ary predicates $\boldsymbol{B}_{1}\left(\right.$ ) and $\boldsymbol{B}_{2}($ ).

(2) The individual constants are $1,2,3,4, \ldots, n, \ldots$

(3) The individual variables are $x_{1}, x_{2}, \ldots, x_{i}, \ldots$ The predicate (set) variables are $X_{1}, X_{2}, \ldots, X_{i}, \ldots$ 
(4) The well-formed formulas of $\mathfrak{L}_{\mathfrak{A}}$ are defined as follows:

(a) If $\alpha$ and $\beta$ are individual constants or variables then $\alpha=\beta$ is a wff.

(b) If $\psi()$ is an n-place predicate constant or predicate variable, and $\alpha_{1}, \ldots, \alpha_{n}$ are individual variables or constants, then $\psi\left(\alpha_{1} \cdots \alpha_{n}\right)$ is a wff.

(c) If $\psi$ and $\varphi$ are wff's then so are $\psi \wedge \varphi$ and $\neg \psi$.

(d) If $x_{i}$ is free in the wff $\varphi$, then $\left(x_{i}\right) \varphi$ is a wff; if $X_{i}$ is free in the wff $\varphi$, then $\left(X_{i}\right) \varphi$ is a wff.

(e) Nothing is a wff except by virtue of (a)-(d).

Definition 6.4. Suppose we have some $\mathfrak{A} \subset 2^{N}$ and two sets $B_{1}, B_{2} \subset N$, then for $\mathfrak{R}=\left\langle N ; \mathfrak{A} ;+; \times ; B_{1} ; B_{2} ; \ldots, n, \ldots ; \ldots, a, \ldots\right\rangle$ we define the satisfaction relation $\mathfrak{R}(\mathrm{SAT}) \varphi$ between $\mathfrak{R}$ and sentences $\varphi$ of $\mathfrak{R}_{\mathfrak{A}}$, where the individual constants $0,1,2, \ldots$ stand for the appropriate integer, the predicate constants + and $\times$ are given their standard interpretation, the constant $a(\quad)$, for $a \in \mathfrak{A}$, holds of exactly the members of $a, B_{1}()$, and $B_{2}(\quad)$ hold of exactly the members of $B_{1}$ and $B_{2}$ respectively. The quantifiers are interpreted so that $x_{1}, x_{2}, \ldots$ range over $N$ and $X_{1}, X_{2}, \ldots$, range over the sets in $\mathfrak{A}$. [Note: This means that if $B_{1}$, for example, is not an element of $\mathfrak{A}$, then it is not in the range of the quantifiers $X_{i}$.] We leave it to the reader to formalize the definition of (SAT).

Definition 6.5. In the rest of this section we are interested in countable $\mathfrak{A} \subset 2^{N}$ with the following property $\mathfrak{B}:(n \in N)(\exists a, b \in \mathfrak{A})[n \in a \wedge n \notin b]$.

Suppose now that $\mathfrak{A}$ is some countable subset of $2^{N}$ satisfying property $\mathfrak{B}$.

DEFINITION 6.6. A condition $P$ is a pair $\left\langle s_{P}, t_{P}\right\rangle$ of finite sequences of elements of A. We write

$$
s_{P}=\left\langle S_{0}^{P}, S_{1}^{P}, \ldots, S_{n_{P}}^{P}\right\rangle \quad t_{P}=\left\langle T_{0}^{P}, T_{1}^{P}, \ldots, T_{m_{P}}^{P}\right\rangle
$$

and we write $P \subset P^{\prime}$ just in case $s_{P^{\prime}}$ extends $s_{P}$, and $t_{P^{\prime}}$ extends $t_{P}$.

DEFINITION 6.7. Let

$$
\tau: N \times N \underset{\text { onto }}{\stackrel{1-1}{\longrightarrow}} N
$$

be any standard recursive coding function, $\pi_{1}(\tau(x, y))=x, \pi_{2}(\tau(x, y))=y$. We define a relation $P(\mathrm{FOR}) \varphi$ (forcing) between conditions $P$ and sentences $\varphi$ of $\mathfrak{Q}_{\mathfrak{U}}$ as follows:

(i) If $\varphi$ is $+(\boldsymbol{m}, \boldsymbol{n}, \boldsymbol{r}), \times(\boldsymbol{m}, \boldsymbol{n}, \boldsymbol{r})$, or $\boldsymbol{m}=\boldsymbol{n}$, then $P(\mathrm{FOR}) \varphi$ just in case $\varphi$ is true in arithmetic.

(ii) If $\varphi$ is $a(\boldsymbol{n})$ for some $a \in \mathfrak{A}$, then $P(\mathrm{SAT}) \varphi$ just in case $n \in a$.

(iii) If $\varphi$ is $B_{1}(n)$, let $x=\pi_{1}(n), y=\pi_{2}(n)$, then $P(\mathrm{FOR}) \varphi$ iff $n_{P} \geqq y$ and $x \in S_{y}^{P}$.

(iv) If $\varphi$ is $\boldsymbol{B}_{2}(n)$, let $x=\pi_{1}(n), y=\pi_{2}(n)$, then $P(\mathrm{FOR}) \varphi$ iff $m_{P} \geqq y$ and $x \in T_{y}^{P}$.

(v) If $\varphi$ is $\psi \wedge \chi$, then $P$ (FOR) $\varphi$ iff $P$ (FOR) $\psi$ and $P$ (FOR) $\chi$.

(vi) If $\varphi$ is $\neg \psi$, then $P(\mathrm{FOR}) \varphi$ iff $\left(P^{\prime} \supset P\right)\left[\sim P^{\prime}(\mathrm{FOR}) \psi\right]$.

(vii) If $\varphi$ is $\left(x_{i}\right) \psi$, then $P(\mathrm{FOR}) \varphi$ iff $(n \in N)[P(\mathrm{FOR}) \psi(n)]$.

(viii) If $\varphi$ is $\left(X_{i}\right) \psi$, then $P(\mathrm{FOR}) \varphi$ iff $(a \in \mathfrak{U})[P(\mathrm{FOR}) \psi(a)]$.

Most of the following lemmas are standard in any development of forcing, and follow easily by induction on the length of the relevant formulas. In many cases we 
abbreviate the proof or omit it entirely. Lemmas peculiar to this notion of forcing, or to our particular application are marked with an asterisk $\left(^{*}\right)$.

LEMMA 6.8. “(FOR)" is well defined by (i)-(viii).

LEMMA 6.9. It never happens that $P(\mathrm{FOR}) \varphi$ and $P(\mathrm{FOR}) \neg \varphi$.

Lemma 6.10. If $P(\mathrm{FOR}) \varphi$ and $P^{\prime} \supset P$, then $P^{\prime}(\mathrm{FOR}) \varphi$.

The proof is by induction on the length of $\varphi$.

LEMMA 6.11. (a) If $P(\mathrm{FOR}) \varphi$ and $P^{\prime} \supset P$, then $\sim P^{\prime}(\mathrm{FOR}) \neg \varphi$.

(b) If $P(\mathrm{FOR}) \neg \varphi$ and $P^{\prime} \supset P$, then $\sim P^{\prime}(\mathrm{FOR}) \varphi$.

This is immediate from Lemmas 6.9 and 6.10.

*Lemma 6.12. For all $P, \varphi$, if $\sim P(\mathrm{FOR}) \varphi$ then $\left(\exists P^{\prime} \supset P\right)\left[P^{\prime}(\mathrm{FOR}) \neg \varphi\right]$.

The proof is easy by induction on the length of $\varphi$, but we need the property $\mathfrak{P}$ of $\mathfrak{U}$ to prove the result for atomic sentences.

LEMMA 6.13. $P(\mathrm{FOR}) \varphi$ iff $P(\mathrm{FOR}) \neg \neg \varphi$.

This is immediate from Lemma 6.12 and clause (vi) of the definition of (FOR).

Lemma 6.14. (a) Suppose $P(\mathrm{FOR}) \neg\left(x_{i}\right) \varphi$, then $\left(\exists P^{\prime} \supset P\right)(\exists n)\left[P^{\prime}(\mathrm{FOR}) \neg \varphi(n)\right]$.

(b) Suppose $P(\mathrm{FOR}) \neg\left(X_{i}\right) \varphi$, then $\left(\exists P^{\prime} \supset P\right)(\exists a \in \mathfrak{U})\left[P^{\prime}(\mathrm{FOR}) \neg \varphi(a)\right]$.

Proof. (a) Suppose $P($ FOR $) \neg\left(x_{i}\right) \varphi$, then no extension of $P$ forces $\left(x_{i}\right) \varphi$; in particular, $\sim P(\mathrm{FOR})\left(x_{i}\right) \varphi$. But then for some $n_{0}, \sim P(\mathrm{FOR}) \varphi\left(n_{0}\right)$, so by Lemma 6.12 there is some $P^{\prime} \supset P$ such that $P^{\prime}(\mathrm{FOR}) \neg \varphi\left(n_{0}\right)$.

(b) Completely analogous.

*Lemma 6.15. (a) Suppose $\varphi \in \mathfrak{Q}_{\mathfrak{X}}$ does not involve $\boldsymbol{B}_{2}$, then for all conditions $\langle s, t\rangle,\langle s, t\rangle$ (FOR) $\varphi$ iff for all $t^{\prime},\left\langle s, t^{\prime}\right\rangle$ (FOR) $\varphi$.

(b) Suppose $\varphi \in \mathfrak{Q}_{\mathfrak{Q}}$ does not involve $\boldsymbol{B}_{1}$, then for all conditions $\langle s, t\rangle,\langle s, t\rangle(\mathrm{FOR}) \varphi$ iff for all $s^{\prime},\left\langle s^{\prime}, t\right\rangle$ (FOR) $\varphi$.

The result is obvious; it follows by induction on the length of $\varphi$.

DEFINITION 6.16. A sequence $\left\{P_{i}\right\}_{i \in N}$ of conditions is complete iff:

(i) for all $i, P_{i} \subset P_{i+1}$, and

(ii) for all sentences $\varphi \in \mathfrak{L}_{\mathfrak{U}}$ there exists some $i$ such that $P_{i}(\mathrm{FOR}) \varphi$ or $P_{i}(\mathrm{FOR}) \neg \varphi$, and

(iii) (a) if for some $i, P_{i}$ (FOR) $\neg\left(x_{j}\right) \varphi\left(x_{j}\right)$, then there are $k, n$ such that $P_{k}$ (FOR) $\neg \varphi(\boldsymbol{n})$, and

(b) if for some $i, P_{i}(\mathrm{FOR}) \neg\left(X_{i}\right) \varphi\left(X_{i}\right)$, then there are $k \in N$ and $a \in \mathfrak{A}$ such that $P_{k}(\mathrm{FOR}) \neg \varphi(\boldsymbol{a})$.

LEMMA 6.17. Let $P$ be any condition, then there is a complete sequence $\left\{P_{i}\right\}$, with $P_{0}=P$. 
This follows from the countability of $\mathfrak{Q}_{\mathfrak{Q}}$ together with Lemmas 6.12 and 6.14.

Definition 6.18. If $P$ is a condition then we define two sets $B_{1}^{P}$ and $B_{2}^{P}$ as follows:

$$
\begin{array}{lll}
\tau(x, y) \in B_{1}^{P} \Leftrightarrow y \leqq n_{P} & \text { and } & x \in S_{y}^{P}, \\
\tau(x, y) \in B_{2}^{P} \Leftrightarrow y \leqq m_{P} & \text { and } & x \in T_{y}^{P} .
\end{array}
$$

Definition 6.19. If $\left\{P_{i}\right\}$ is a complete sequence, we say that $B_{1}$ and $B_{2}$ are the generic sets associated with $P_{i}$ just in case

$$
B_{1}=\bigcup_{i \in N} B_{1}^{P_{i}}, \quad B_{2}=\bigcup_{i \in N} B_{2}^{P_{i}} .
$$

The model $\mathfrak{N}$ associated with $\left\{P_{i}\right\}$ is

$$
\mathfrak{R}=\left\langle N ; \mathfrak{A} ;+, \times ; B_{1}, B_{2} ; \ldots, n, \ldots ; \ldots, a, \ldots\right\rangle
$$

where $B_{1}$ and $B_{2}$ are the generic sets defined above.

*LemMa 6.20. Let $\left\{P_{i}\right\}$ be a complete sequence of conditions and let $B_{1}$ and $B_{2}$ be the associated generic sets. Then

(a) $(x)\left[x \in B_{1} \Leftrightarrow(\exists i) P_{i}(\mathrm{FOR}) B_{1}(x)\right]$,

(b) $(x)\left[x \in B_{2} \Leftrightarrow(\exists i) P_{i}(\mathrm{FOR}) B_{2}(x)\right]$.

Proof. This is immediate via the definitions of $B_{1}, B_{2}$ and forcing on atomic sentences.

LEMMA 6.21. Let $\left\{P_{i}\right\}$ be a complete sequence of conditions, and let $\mathfrak{N}$ be the associated model. Then for all sentences $\varphi \in \mathfrak{L}_{\mathfrak{A}}, \mathfrak{R}(\mathrm{SAT}) \varphi \Leftrightarrow(\exists i) P_{\mathfrak{i}}(\mathrm{SAT}) \varphi$.

Proof. The lemma follows easily by induction on the length of $\varphi$, provided the following novel definition of length is used:

(i) If $\varphi$ is atomic then $\ln (\varphi)=1$;

(ii) $\ln (\varphi \wedge \psi)=\ln (\varphi)+\ln (\psi)+1$;

(iii) $\ln (\neg \varphi)=\operatorname{lh}(\varphi)+1$;

(iv) (a) $\ln \left(\left(x_{i}\right) \varphi\right)=\operatorname{lh}(\varphi)+2$;

(b) $\ln \left(\left(X_{i}\right) \varphi\right)=\ln (\varphi)+2$.

The effect of this definition is to make $\left(x_{i}\right) \varphi\left(x_{i}\right)$ and $\left(X_{i}\right) \varphi\left(X_{i}\right)$ longer than $\neg \varphi\left(x_{i}\right)$ and $\neg \varphi\left(X_{i}\right)$ respectively. This is exactly the trick needed to make the proof trivial.

*LEMMA 6.22. Let $\left\{P_{i}\right\}$ be a complete sequence of conditions, and $\mathfrak{R}$ the associated model. Suppose $\varphi_{1}(x)$ and $\varphi_{2}(x)$ are formulas with one free variable (a number variable) such that $\varphi_{1}$ does not involve $\boldsymbol{B}_{2}$, and $\varphi_{2}$ does not involve $\boldsymbol{B}_{1}$; and suppose further that

$$
P_{i_{0}}=\left\langle s_{0}, t_{0}\right\rangle(\mathrm{FOR})(x)\left[\varphi_{1}(x) \equiv \varphi_{2}(x)\right]
$$

Let $A=\left\{x \mid \mathfrak{R}(\mathrm{SAT}) \varphi_{1}(x)\right\}$, then $(x)\left[x \in A \Leftrightarrow\left(\exists s^{\prime} \supset s_{0}\right)\left[\left\langle s^{\prime}, \varnothing\right\rangle(\mathrm{FOR}) \varphi_{1}(x)\right]\right]$ where $\varnothing$ is the null sequence from $\mathfrak{A}$. 
Proof. $\Rightarrow$ : Suppose $n \in A$, then by Lemma 6.21 there is some $P_{j} \supset P_{i_{0}}$ such that $P_{j}(\mathrm{FOR}) \varphi_{1}(n)$; let $P_{j}=\left\langle s^{\prime}, t^{\prime}\right\rangle$. By Lemma 6.15 , since $\varphi_{1}$ does not involve $\boldsymbol{B}_{2}$, $\left\langle s^{\prime}, \varnothing\right\rangle(\mathrm{FOR}) \varphi_{1}(n)$, so $s^{\prime}$ is the desired extension of $s_{0}$.

$\Leftarrow$ : Suppose the implication fails in this direction. Then there must be some $s^{\prime}, n$ such that $s^{\prime} \supset s_{0}$ and $\left\langle s^{\prime}, \varnothing\right\rangle(\mathrm{FOR}) \varphi_{1}(n)$ and $n \notin A$. Since $n \notin A$ and $\mathfrak{N}$ (FOR) $(x)\left[\varphi_{1}(x) \equiv \varphi_{2}(x)\right]$ there must be some $P_{j}=\left\langle s^{\prime \prime}, t^{\prime \prime}\right\rangle$ such that $P_{j}(\mathrm{FOR}) \neg \varphi_{2}(n)$ and $P_{i_{0}} \subset P_{j}$. By Lemma $6.15\left\langle s^{\prime}, t^{\prime \prime}\right\rangle($ FOR $) \neg \varphi_{2}(n)$; but $P_{i_{0}} \subset\left\langle s^{\prime}, t^{\prime \prime}\right\rangle$ and $P_{i_{0}}(\mathrm{FOR})$ $(x)\left[\varphi_{1}(x) \equiv \varphi_{2}(x)\right]$. Thus we have it that

$$
\begin{gathered}
\left\langle s^{\prime}, t^{\prime \prime}\right\rangle(\mathrm{FOR}) \text { (i) }(x)\left[\varphi_{1}(x) \equiv \varphi_{2}(x)\right] \\
\text { (ii) } \varphi_{1}(n) \\
\text { (iii) } \neg \varphi_{2}(n),
\end{gathered}
$$

which is impossible. Thus the implication also holds from right to left.

*LEMma 6.23. Let $\left\{P_{i}\right\}$ be any complete sequence of conditions; then both of the associated generic sets $B_{1}$ and $B_{2}$ are uniform upper-bounds for $\mathfrak{A}$.

Proof. It is easy to check that the following sentence (suitably rewritten in $\mathfrak{L}_{\mathfrak{A}}$ ) is forced eventually by every complete sequence of conditions:

$$
(X)(\exists y)(z)\left[X(z) \equiv B_{i}(\tau(z, y))\right] \quad i=1,2 .
$$

We are now prepared to obtain the desired theorem. We now assume that the countable set $\mathfrak{A} \subset 2^{N}$ is such that $\mathfrak{M}=\langle N, \mathfrak{A},+, \times\rangle$ is an $\omega$-model for analysis.

LEMMA 6.24: $\mathfrak{A}$ satisfies property $\mathfrak{P}$. [Hence 6.6-6.23 apply to $\mathfrak{A}$.

Proof. Obvious.

LEMMA 6.25. Suppose $\varphi(x)$ is arithmetical in $x$ and $B_{1}$, and $s_{0}$ is some finite sequence of sets in $\mathfrak{A}$. Then the set

$$
W=\left\{x \mid\left(\exists s^{\prime} \supset s_{0}\right)\left\langle s^{\prime}, \varnothing\right\rangle(\text { FOR }) \varphi(x)\right\}
$$

is first-order definable over $\mathfrak{M}$.

It is easy to show that quantification over finite sequences of elements of $\mathfrak{A}$ is expressible within $\mathfrak{M}$. The result then follows by induction on the length of $\varphi$. It is helpful to take $\varphi$ in prenex normal form.

*Lemma 6.26. Suppose $\left\{P_{i}\right\}$ is a complete sequence and $B_{1}, B_{2}$ are the associated generic sets. If a set $W$ is arithmetical in each of $B_{1}$ and $B_{2}$, then $W \in \mathfrak{A}$.

Proof. Suppose the hypothesis of the lemma obtains; then there are two formulas $\varphi_{1}(x)$ and $\varphi_{2}(x)$, which define $W$ arithmetically in $B_{1}$, and $B_{2}$ respectively. Let $\mathfrak{R}$ be the model associated with $\left\{P_{i}\right\}$, then $\mathfrak{R}(\mathrm{FOR})(x)\left[\varphi_{1}(x) \equiv \varphi_{2}(x)\right]$, so some $P_{j}$ (FOR) $(x)\left[\varphi_{1}(x) \equiv \varphi_{2}(x)\right]$ and the lemma follows from Lemmas 6.22 and 6.25, together with the fact that $\mathfrak{M}=\langle N, \mathfrak{A},+, \times\rangle$ is an $\omega$-model, and is thus closed under first-order definability. 
THEOREM 6.2. Suppose $\mathfrak{M}$ is a countable $\omega$-model for analysis, and $A$ is a uniform upper-bound for $\mathfrak{A}$. Then it cannot be the case that $A$ is arithmetical in every uniform upper-bound for $\mathfrak{A}$.

Proof. Suppose the contrary, and let $A$ be a u.u.b. for $\mathfrak{A}$ which is arithmetical in every u.u.b. for $\mathfrak{A}$. Let $\left\{P_{i}\right\}$ be any complete sequence (for $\mathfrak{Q}_{\mathfrak{A}}$ ) and let $B_{1}, B_{2}$ be the associated generic sets. Then by Lemma $6.23 B_{1}$ and $B_{2}$ are both u.u.b.'s for $\mathfrak{A}$; thus $A$ must be arithmetical in each of them. Then by Lemma 6.26, $A \in \mathfrak{A}$; but this is impossible, because the jump of $A$ would then be a member of $\mathfrak{A}$ (since $\mathfrak{M}$ is an $\omega$-model) and we would have $j(A) \leqq{ }_{T} A$.

Theorem 6.1 (second half of Main Theorem) is an immediate corollary of Theorem 6.2.

7. The R. A. sets are the minimal $\beta$-model for analysis. That the R. A. sets form a countable $\omega$-model for 2-N.T. was proved by Cohen in [C]. Since these sets are just the members of $A_{\beta_{0}}$, it is an immediate corollary of 4.5, 5.3, and 5.4 that this model is a $\beta$-model. [It is immaterial that the formula W.O. $(X)$ in 5.1 is not the usual expression for the property " $X$ is a well-ordering". For the equivalence of $W . O .(X)$ to the usual expression(s) is a theorem of 2-N.T., and hence holds in all $\omega$-models. Since $A_{\beta_{0}}$ is an $\omega$-model, we have, for $X$ in $A_{\beta_{0}}$, that $X$ is a well-ordering $\equiv W . O$. $(X)$ holds in $A_{\beta_{0}} \equiv$ " $X$ is a well-ordering" (i.e., the standard expression for this property) holds in $A_{\beta_{0}}$. But this is just what it means to say that $A_{\beta_{0}}$ is a $\beta$ model.]

In this section, we shall show that this model is minimal in the sense of being the intersection of all $\beta$-models of 2-N.T.

First we need some facts about $\beta$-models of 2-N.T.

To facilitate the statement of these facts, we introduce some notations.

If $B$ is a 2-ary relation-set whose corresponding relation is a well-ordering of integers, we write simply " $B$ is a well-ordering".

If $M$ is a $\beta$-model for analysis, let On $(M)$ be the least ordinal $\alpha$ such that there is no well-ordering of order-type $\alpha$ in $M$, and if $B$ is a well-ordering, let $|B|$ be its order-type.

Henceforth, let $M$ be a special variable for $\beta$-models.

Our first lemma is left to the reader to verify.

LEMMA 7.1. On $(M)$ is a limit number.

The need for the next lemma will become obvious shortly.

LEMMA 7.2. If $S$ is a set in $M$, then there are well-orderings in $M$ of all the following order-types: $\omega_{1}^{(n), s}$ for $n=0,1,2, \ldots$

The proof is left to the reader. It is necessary to use the fact that $\Pi_{1}^{1}$-in- $S$ notions are "absolute" in $\beta$-models containing $S$.

LEMMA 7.3. On $(M)$ is HYP. 
Proof. By Lemma 7.2 (taking $S=\varnothing$ ), $\omega_{1}^{\infty} \leqq$ On $(M)$. By Lemma 4.2, either On $(M)$ is HYP, or there is a greatest ordinal $\beta<$ On $(M)$ such that $\beta$ is HYP. In the latter case, there are two subcases.

SUBCASE 1. $\beta_{0} \leqq \beta$. Then $A_{\text {On(M) }}=A_{\beta}=A_{\beta_{0}}$ (since $A_{\gamma}=A_{\beta_{0}}$ for all $\gamma>\beta_{0}$ ).

But $A_{\beta_{0}}$ is closed under hyperjump. So $A_{\mathrm{On}(M)}$ is closed under hyperjump-i.e. On $(M)$ is HYP.

SubCASE 2. $\beta<\beta_{0}$. Then, by Lemmas 3.11 and 4.3 , there is a set $S$ in $A_{\beta+1}$ such that $\omega_{1}^{S}>\beta$. By Corollary $3.7, \omega_{1}^{\infty, s}$ is the least HYP ordinal greater than $\beta$. Let $A$ be a well-ordering in $M$ of order type $\beta+1$ (such must exist, since $\beta$, and hence $\beta+1$, is less than On $(M)$ ). All sets in $A_{\beta+1}$ are hyperarithmetical in $A$, by Lemma 2.7 . Since all $\beta$-models are closed under "hyperarithmetical in", it follows that $S$ is in $M$. Thus by $7.2 \omega_{1}^{\infty, s}$ is a HYP ordinal $\leqq$ On $(M)$, and, since $\beta$ is the largest HYP ordinal $<$ On $(M)$, it follows that $\omega_{1}^{\infty, s}=$ On $(M)$, i.e., On $(M)$ is HYP.

Let $R A(X)$ be the formula

$$
(\exists Z)(\exists W)\left[W . O .(Z) \wedge \operatorname{CONSTR}(Z, W) \wedge(\exists x, y)\left(X={ }_{w} S_{y}^{x}\right)\right] .
$$

Lemma 7.4. For all $X$ in $M, M(\mathrm{SAT}) R A(X)$ if and only $X \in A_{\mathrm{On}(M)} \subset M$.

This is straightforward to verify by examining the formula $R A(X)$ and using the facts that (1) $M$ contains well-orderings of all (and only) lengths $<$ On $(M)$; and (2) $M$ is a $\beta$-model.

The next lemma is the crucial one.

LEMMA 7.5. On $(M) \geqq \beta_{0}$.

Proof. Suppose the contrary. Then, since On $(M)$ is HYP by Lemma 7.3, and assumed to be $<\beta_{0}$, it follows from Lemma 4.3 that there is a well-ordering of order-type On $(M)$ definable over $A_{\text {On( }(M)}$ by a formula (say $\varphi(x, y)$ ) of 2-N.T. Let $\varphi^{*}(x, y)$ be the formula that results if each 2 nd order quantifier in $\varphi(x, y)$ is relativized to $R A(X)$.

By Lemma 7.4, $M(\operatorname{SAT}) \varphi^{*}(n, m)$ if and only if $A_{\mathrm{On}(M)}(\mathrm{SAT}) \varphi(n, m)$ (for all $n$, $m \in \omega)$.

But this says that the formula $\varphi^{*}(x, y)$ defines over $M$ just the relation that $\varphi(x, y)$ defines over $A_{\mathrm{On}(M)}$, and this relation is a well-ordering of order-type $\mathrm{On}(M)$. Thus there is a well-ordering of order-type On $(M)$ in $M$ (since all sets definable over $M$ are also in $M$, by the property of being a model of 2-N.T.), contrary to the property of On $(M)$. Contradiction.

THEOREM 7.6. The R.A. sets are the minimal $\beta$-model for 2-N.T.

Proof. We have already observed that $A_{\beta_{0}}$ (i.e., the R.A. sets) is a $\beta$-model for 2-N.T. That $A_{\beta_{0}}$ is a subset of $M$, where $M$ is an arbitrary $\beta$-model, is an immediate consequence of Lemmas 7.4 and 7.5. 


\title{
REFERENCES
}

[C] P. J. Cohen, A minimal model for set theory, Bull. Amer. Math. Soc. 69 (1963), 537-540.

[D] M. Davis, Computability and unsolvability, McGraw-Hill, New York, 1958.

[E] H. B. Enderton, Hierarchies in recursive function theory, Trans. Amer. Math. Soc. 111 (1964), 457-471.

[GP] R. Gandy and H. Putnam, A minimal $\beta$-model for analysis, (to appear).

[G] K. Gödel, The consistency of the continuum hypothesis, Princeton Univ. Press, Princeton, N.J., 1940 (fourth printing, 1958).

[HP] G. Hensel and H. Putnam, On the notational independence of various hierarchies of degrees of unsolvability, J. Symbolic Logic 30 (1965), 69-86.

[K] S. C. Kleene, Quantification of number-theoretic predicates, Compositio Math. 14 (1959), 23-40.

[K'] - Arithmetical predicates and function quantifiers, Trans. Amer. Math. Soc. 79 (1955), 312-340.

[S] C. Spector, Recursive well orderings, J. Symbolic Logic 20 (1955), 151-163.

\author{
HARVARD UNIVERSITY, \\ Cambridge, MassachusetTs \\ Catholic University, \\ WASHINGTON, D.C.
}

